\title{
NONLINEAR ROBUST TRACKING CONTROL OF A QUADROTOR UAV ON SE(3)
}

\author{
Taeyoung Lee, Melvin Leok, and N. Harris McClamroch
}

\begin{abstract}
This paper provides nonlinear tracking control systems for a quadrotor unmanned aerial vehicle (UAV) that are robust to bounded uncertainties. A mathematical model of a quadrotor UAV is defined on the special Euclidean group, and nonlinear output-tracking controllers are developed to follow (i) an attitude command, and (ii) a position command for the vehicle center of mass. The controlled system has the desirable properties that the tracking errors are uniformly ultimately bounded, and the size of the ultimate bound can be reduced arbitrarily by control system parameters. Numerical examples illustrating complex maneuvers are provided.
\end{abstract}

Key Words: Quadrotor UAV, geometric Control, special Euclidean group

\section{INTRODUCTION}

A quadrotor unmanned aerial vehicle (UAV) consists of two pairs of counter-rotating rotors and propellers. It has been envisaged for various applications such as surveillance or mobile sensor networks as well as for educational purposes, and several control systems have been studied.

Linear control systems have been widely used to enhance the stability properties of an equilibrium of a quadrotor UAV [1-3]. Several nonlinear controllers have been developed as well. Backstepping and sliding mode techniques are applied in $[4,5]$, and a nonlinear $H_{\infty}$ controller is studied in [6]. An adaptive neural network based control system is developed in [7].

Since all of these controllers are based on Euler angles, they exhibit singularities when representing complex rotational maneuvers of a quadrotor UAV, thereby significantly restricting their ability to achieve complex flight maneuvers.

An attitude control system based on quaternions is applied to a quadrotor UAV [8]. Quaternions do not have singularities, but they have ambiguities in representing an attitude, as the three-sphere $\mathrm{S}^{3}$ double-covers $\mathrm{SO}(3)$. As a result, in a quaternion-based attitude control system, convergence to a single attitude implies convergence to either of the two disconnected, antipodal points on $S^{3}$ [9]. The ambiguity

Manuscript received April 26, 2011; revised October 27, 2011; accepted May 3, 2012.

Taeyoung Lee is with Mechanical and Aerospace Engineering, The George Washington University, Washington, DC 20052, USA (corresponding author, e-mail: tylee@gwu.edu).

Melvin Leok is with Mathematics, University of California at San Diego, La Jolla, CA 92093, USA (e-mail: mleok@ucsd.edu).

N. Harris McClamroch is with Aerospace Engineering, University of Michigan, Ann Arbor, MI 48109, USA (e-mail: nhm@umich.edu).

This work has been supported by by NSF under grants CMMI-1029445, CMMI1029551, DMS-0726263, DMS-100152 and DMS-1010687. in representing an attitude should be carefully resolved in any quaternion-based attitude control system. Otherwise, it may become sensitive to small measurement noises [10], or it may also exhibit unwinding behavior, where the controller rotates a rigid body through unnecessarily large angles [11, 12].

Attitude control systems also have been developed directly on the special orthogonal group, $\mathrm{SO}(3)$, to avoid the singularities associated with Euler angles and the ambiguity of quaternions [13-16]. By following this geometric approach, the dynamics of a quadrotor UAV is globally expressed on the special Euclidean group, SE(3), and nonlinear control systems are developed to track outputs of several flight modes, namely an attitude controlled flight mode, a position controlled flight mode, and a velocity controlled flight mode [17, 18]. Several aggressive maneuvers of a quadrotor UAV are also demonstrated based on a hybrid control architecture.

In this paper, we extend the results of $[17,18]$ to construct nonlinear robust tracking control systems on $\mathrm{SE}(3)$ for a quadrotor UAV. We assume that there exist unstructured, bounded uncertainties, with pre-determined bounds, on the translational dynamics and the rotation dynamics of a quadrotor UAV. Output tracking control systems are developed to follow an attitude command or a position command for the vehicle center of mass. We show that the tracking errors are uniformly ultimately bounded, and the size of the ultimate bound can be reduced arbitrarily. Robustness of the proposed tracking control systems is critical in generating complex maneuvers, as the impact of several aerodynamic effects arising from the variation in airspeed is significant even at moderate velocities [2].

Tracking control of a quadrotor UAV has been considered in $[19,20]$, but the control system in [19] has a complex structure since it is based on a multiple-loop backstepping 
approach, and no stability proof is presented in [20]. Recently, robust tracking control systems are studied in [21, 22], but the quadrotor dynamics simplified by considering planar motion only [21], or by ignoring the rotational dynamics using a timescale separation assumption [22]. Aggressive maneuvers are demonstrated in [23], but are based on Eulerangles. Compared with these results, the unique features of the control system proposed in this paper are as follows: (i) it is developed for the full six degrees of freedom dynamic model of a quadrotor UAV on SE(3); (ii) a rigorous Lyapunov analysis, that explicitly considers the coupling between the translational dynamics and the rotational dynamics, is presented to establish stability properties; and (iii) it is robust against unstructured uncertainties in both the translational dynamics and the rotational dynamics of a quadrotor UAV.

In [24], the quadrotor dynamics are modeled as a collection of simplified hybrid dynamic modes, and reachability sets are analyzed to guarantee the safety and performance for a larger area of operating conditions. In contrast, another advantage of the proposed control system is that complicated reachability set analysis is not required to guarantee safe switching between different flight modes, as the region of attraction for each flight mode covers the configuration space almost globally.

The paper is organized as follows. We develop a globally defined model for the translational and rotational dynamics of a quadrotor UAV in Section II. A hybrid control architecture is introduced in Section III and a robust attitude tracking control system is developed in Section IV. Section V presents results for a robust position tracking, followed by numerical examples in Section VI.

\section{QUADROTOR DYNAMICS MODEL}

Consider a quadrotor UAV model illustrated in Fig. 1. This is a system of four identical rotors and propellers located at the vertices of a square, which generate a thrust and torque normal to the plane of this square. We choose an inertial reference frame $\left\{\vec{e}_{1}, \vec{e}_{2}, \vec{e}_{3}\right\}$ and a body-fixed frame $\left\{\vec{b}_{1}, \vec{b}_{2}, \vec{b}_{3}\right\}$. The origin of the body-fixed frame is located at the center of mass of this vehicle. The first and the second axes of the body-fixed frame, $\vec{b}_{1}, \vec{b}_{2}$, lie in the plane defined by the centers of the four rotors, as illustrated in Fig. 1. The third body-fixed axis $\vec{b}_{3}$ is normal to this plane. Each of the inertial reference frame and the body-fixed reference frame consist of a triad of orthogonal vectors defined according to the right-hand rule. Define:

\footnotetext{
$m \in \mathbb{R} \quad$ the total mass

$J \in \mathbb{R}^{3 \times 3} \quad$ the inertia matrix with respect to the bodyfixed frame

$R \in \mathrm{SO}(3)$ the rotation matrix from the body-fixed frame to the inertial frame
}

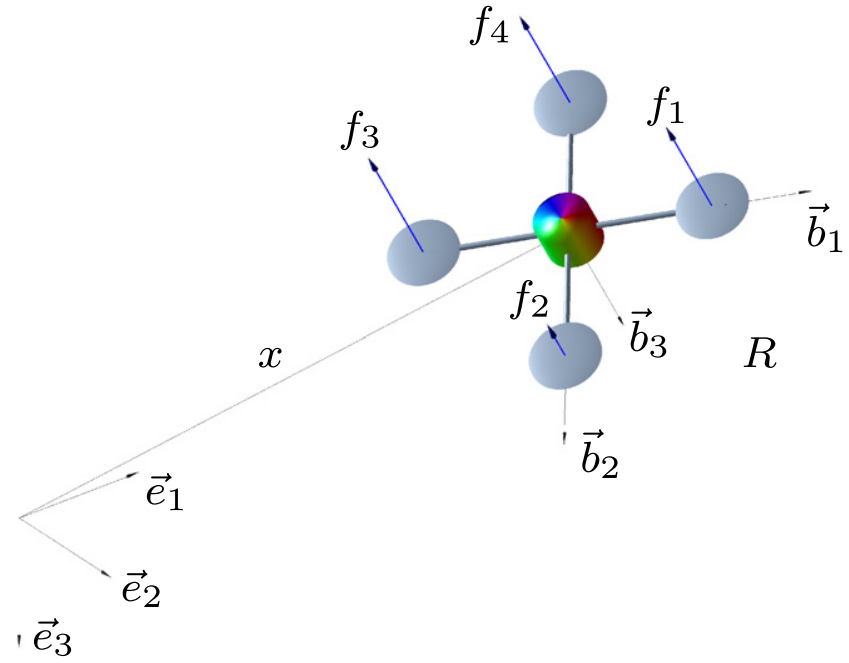

Fig. 1. Quadrotor model.

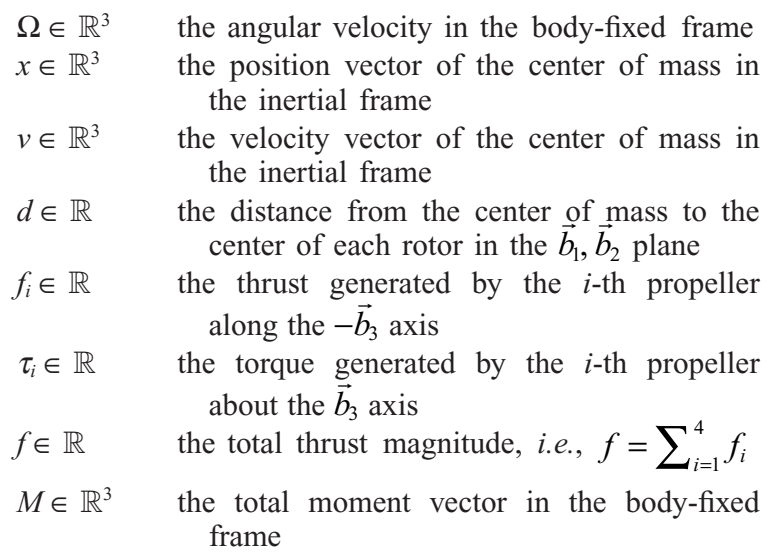

The configuration of this quadrotor UAV is defined by the location of the center of mass and the attitude with respect to the inertial frame. Therefore, the configuration manifold is the special Euclidean group $\mathrm{SE}(3)$, which is the semidirect product of $\mathbb{R}^{3}$ and the special orthogonal group $\mathrm{SO}(3)=\left\{R \in \mathbb{R}^{3 \times 3} \mid R^{T} R=I\right.$, det $\left.R=1\right\}$.

The following conventions are assumed for the rotors and propellers, and the thrust and moment that they exert on the quadrotor UAV. We assume that the thrust of each propeller is directly controlled, and the direction of the thrust of each propeller is normal to the quadrotor plane. The first and third propellers are assumed to generate a thrust along the direction of $-\vec{b}_{3}$ when rotating clockwise; the second and fourth propellers are assumed to generate a thrust along the same direction of $-\vec{b}_{3}$ when rotating counterclockwise. Thus, the thrust magnitude is $f=\sum_{i=1}^{4} f_{i}$, and it is positive when the total thrust vector acts along $-\vec{b}_{3}$, and it is negative when the total thrust vector acts along $\vec{b}_{3}$. By the definition of the rotation matrix $R \in \mathrm{SO}(3)$, the total thrust vector is given by 
$-f R e_{3} \in \mathbb{R}^{3}$ in the inertial frame. We also assume that the torque generated by each propeller is directly proportional to its thrust. Since it is assumed that the first and the third propellers rotate clockwise and the second and the fourth propellers rotate counterclockwise to generate a positive thrust along the direction of $-\vec{b}_{3}$, the torque generated by the $i$-th propeller about $\vec{b}_{3}$ can be written as $\tau_{i}=(-1)^{i} c_{\tau f} f_{i}$ for a fixed constant $c_{\tau f}$. All of these assumptions are common $[3,8]$.

Under these assumptions, the moment vector in the body-fixed frame is given by

$$
\left[\begin{array}{c}
f \\
M_{1} \\
M_{2} \\
M_{3}
\end{array}\right]=\left[\begin{array}{cccc}
1 & 1 & 1 & 1 \\
0 & -d & 0 & d \\
d & 0 & -d & 0 \\
-c_{\tau f} & c_{\tau f} & -c_{\tau f} & c_{\tau f}
\end{array}\right]\left[\begin{array}{l}
f_{1} \\
f_{2} \\
f_{3} \\
f_{4}
\end{array}\right] .
$$

The determinant of the above $4 \times 4$ matrix is $8 c_{\tau f} d^{2}$, so it is invertible when $d \neq 0$ and $c_{\tau f} \neq 0$. Therefore, for given thrust magnitude $f$ and given moment vector $M$, the thrust of each propeller $f_{1}, f_{2}, f_{3}, f_{4}$ can be obtained from (1). Using this equation, the thrust magnitude $f \in \mathbb{R}$ and the moment vector $M \in \mathbb{R}^{3}$ are viewed as control inputs in this paper.

The equations of motion of the quadrotor UAV can be written as

$$
\begin{aligned}
& \dot{x}=v, \\
& m \dot{v}=m g e_{3}-f R e_{3}+\Delta_{x}, \\
& \dot{R}=R \hat{\Omega}, \\
& J \dot{\Omega}+\Omega \times J \Omega=M+\Delta_{R},
\end{aligned}
$$

where the hat map $\hat{\therefore}: \mathbb{R}^{3} \rightarrow \mathfrak{s o}(3)$ is defined by the condition that $\hat{x} y=x \times y$ for all $x, y \in \mathbb{R}^{3}$. (see Appendix 1.1). The inverse of the hat map is denoted by the vee map, $\mathrm{V}: \mathfrak{s o}(3) \rightarrow \mathbb{R}^{3}$.

Unstructured uncertainties in the translational dynamics and the rotational dynamics of a quadrotor UAV are denoted by $\Delta_{x}$ and $\Delta_{R} \in \mathbb{R}^{3}$, respectively. We assume that uncertainties are bounded:

$$
\left\|\Delta_{x}\right\| \leq \delta_{x}, \quad\left\|\Delta_{R}\right\| \leq \delta_{R}
$$

for known, positive constants $\delta_{x}, \delta_{R} \in \mathbb{R}$. Therefore, this paper considers only the bounded and additive uncertainties in the translational dynamics, and the rotational dynamics. It is assumed that the state variables $(x, v, R, \Omega)$ are available for control systems.

Throughout this paper, $\lambda_{m}(\cdot)$ and $\lambda_{M}(\cdot)$ denote the minimum eigenvalue and the maximum eigenvalue of a matrix, respectively. The dot product of two vectors is denoted by $x \cdot y=x^{T} y$ for $x, y \in \mathbb{R}^{3}$.

\section{GEOMETRIC TRACKING CONTROLS}

Since the quadrotor UAV has four inputs, it is possible to achieve asymptotic output tracking for, at most, four quadrotor UAV outputs. The quadrotor UAV has three translational and three rotational degrees of freedom; it is not possible to achieve asymptotic output tracking of both the attitude and position of the quadrotor UAV simultaneously. This motivates us to introduce several flight modes. Each flight mode is characterized by the exact tracking of a specified set of outputs.

The three flight modes considered in this paper are:

- Attitude controlled flight mode: the outputs are the attitude of the quadrotor UAV and the controller for this flight mode achieves asymptotic attitude tracking.

- Position controlled flight mode: the outputs are the position vector of the center of mass of the quadrotor UAV and the controller for this flight mode achieves asymptotic position tracking.

- Velocity controlled flight mode: the outputs are the velocity vector of the center of mass of the quadrotor UAV and the controller for this flight mode achieves asymptotic velocity tracking.

A complex flight maneuver can be defined by specifying a concatenation of flight modes together with conditions for switching between them; for each flight mode one also specifies the desired or commanded outputs as functions of time. For example, one might define a complex aerobatic flight maneuver for the quadrotor UAV that consists of a hovering flight segment by specifying a constant position vector, a reorientation segment by specifying the time evolution of the vehicle attitude, and a surveillance flight segment by specifying a time-varying position vector. The controller in such a case would switch between nonlinear controllers defined for each of the flight modes.

These types of complex aerobatic maneuvers, involving large angle transitions between flight modes, have not been much studied in the literature. Such hybrid flight control architectures have been proposed in [24-27], but they are sensitive to switching conditions due to a limited region of attraction for each flight mode; they require complicated reachability set analyses to guarantee safety and performance. The proposed control system is robust to switching conditions since each flight mode has almost global stability properties, making design of a complex quadrotor maneuver straightforward.

This work is also distinct from motion planning results in [28], where an algorithm to solve an open-loop motion 
planning problem is proposed; a complex trajectory is obtained by joining a finite number of specific maneuvers, referred to as motion primitives. This paper illustrates that a complex maneuver can be achieved by concatenating several flight modes, where the desired trajectory for each flight mode can be chosen arbitrarily.

\section{ATTITUDE CONTROLLED FLIGHT MODE}

In this section, an attitude controlled flight mode is considered, where the outputs are the attitude of the quadrotor UAV and the controller for this flight mode achieves asymptotic attitude tracking.

\subsection{Attitude tracking errors}

Suppose that an arbitrary smooth attitude command $R_{d}(t) \in \mathrm{SO}(3)$ is given. The corresponding angular velocity command is obtained by the attitude kinematics equation, $\hat{\Omega}_{d}=R_{d}^{T} \dot{R}_{d}$.

We first define errors associated with the attitude dynamics of the quadrotor UAV. The attitude error function studied in $[13,29,30]$, and several properties are summarized as follows.

Proposition 1. For a given tracking command $\left(R_{d}, \Omega_{d}\right)$, and the current attitude and angular velocity $(R, \Omega)$, we define an attitude error function $\Psi: \mathrm{SO}(3) \times \mathrm{SO}(3) \rightarrow \mathbb{R}$, an attitude error vector $e_{R} \in \mathbb{R}^{3}$, and an angular velocity error vector $e_{\Omega} \in \mathbb{R}^{3}$ as follows:

$$
\begin{aligned}
& \Psi\left(R, R_{d}\right)=\frac{1}{2} \operatorname{tr}\left[I-R_{d}^{T} R\right], \\
& e_{R}=\frac{1}{2}\left(R_{d}^{T} R-R^{T} R_{d}\right)^{\vee}, \\
& e_{\Omega}=\Omega-R^{T} R_{d} \Omega_{d},
\end{aligned}
$$

Then, the following statements hold:

(i) $\Psi$ is locally positive-definite about $R=R_{d}$.

(ii) the left-trivialized derivative of $\Psi$ is given by

$$
\mathrm{T}_{I}^{*} \mathrm{~L}_{R}\left(\mathbf{D}_{R} \Psi\left(R, R_{d}\right)\right)=e_{R} .
$$

(iii) the critical points of $\Psi$, where $e_{R}=0$, are $\left\{R_{d}\right\} \cup$ $\left\{R_{d} \exp (\pi \hat{s}), s \in \mathrm{S}^{2}\right\}$.

(iv) a lower bound of $\Psi$ is given as follows:

$$
\frac{1}{2}\left\|e_{R}\left(R, R_{d}\right)\right\|^{2} \leq \Psi\left(R, R_{d}\right),
$$

(v) Let $\psi$ be a positive constant that is strictly less than 2 . If $\Psi\left(R, R_{d}\right)<\psi<2$, then an upper bound of $\Psi$ is given by

$$
\Psi\left(R, R_{d}\right) \leq \frac{1}{2-\psi}\left\|e_{R}\left(R, R_{d}\right)\right\|^{2} .
$$

Proof. See [30].

\subsection{Attitude tracking controller}

We now introduce a nonlinear controller for the attitude controlled flight mode, described by an expression for the moment vector:

$$
\begin{aligned}
M= & -k_{R} e_{R}-k_{\Omega} e_{\Omega}+\Omega \times J \Omega \\
& -J\left(\hat{\Omega} R^{T} R_{d} \Omega_{d}-R^{T} R_{d} \dot{\Omega}_{d}\right)+\mu_{R}, \\
\mu_{R}= & -\frac{\delta_{R}^{2} e_{A}}{\delta_{R}\left\|e_{A}\right\|+\varepsilon_{R}}, \\
e_{A}= & e_{\Omega}+c_{2} J^{-1} e_{R},
\end{aligned}
$$

where $k_{R}, k_{\Omega}, c_{2}, \varepsilon_{R}$ are positive constants.

In this attitude controlled mode, it is possible to ignore the translational motion of the quadrotor UAV; consequently the reduced model for the attitude dynamics are given by (4), (5), using the controller expression (13)-(15). We now state the result that the tracking errors $\left(e_{R}, e_{\Omega}\right)$ are uniformly ultimately bounded.

Proposition 2. (Robustness of Attitude Controlled Flight Mode) Suppose that the initial attitude error satisfies

$$
\Psi\left(R(0), R_{d}(0)\right)<\psi_{2}<2
$$

for a constant $\psi_{2}$. Consider the control moment $M$ defined in (13)-(15). For positive constants $k_{R}, k_{\Omega}$, the constants $c_{2}, \varepsilon_{R}$ are chosen such that

$$
\begin{aligned}
& c_{2}<\min \left\{\frac{4 k_{\Omega} k_{R} \lambda_{m}(J)^{2}}{k_{\Omega}^{2} \lambda_{M}(J)+4 k_{R} \lambda_{m}(J)^{2}}, \sqrt{k_{R} \lambda_{m}(J)}\right\}, \\
& \varepsilon_{R}<\frac{\lambda_{m}\left(M_{21}\right) \lambda_{m}\left(W_{2}\right)}{\lambda_{M}\left(M_{22}\right)} \psi_{2}\left(2-\psi_{2}\right),
\end{aligned}
$$

where the matrices $M_{21}, M_{22}, W_{2} \in \mathbb{R}^{2 \times 2}$ are given by

$$
M_{21}=\frac{1}{2}\left[\begin{array}{cc}
k_{R} & -c_{2} \\
-c_{2} & \lambda_{m}(J)
\end{array}\right], M_{22}=\frac{1}{2}\left[\begin{array}{cc}
\frac{2 k_{R}}{2-\psi_{2}} & c_{2} \\
c_{2} & \lambda_{M}(J)
\end{array}\right],
$$




$$
W_{2}=\left[\begin{array}{cc}
\frac{c_{2} k_{R}}{\lambda_{M}(J)} & -\frac{c_{2} k_{\Omega}}{2 \lambda_{m}(J)} \\
-\frac{c_{2} k_{\Omega}}{2 \lambda_{m}(J)} & k_{\Omega}-c_{2}
\end{array}\right] .
$$

Then, the attitude tracking errors $\left(e_{R}, e_{\Omega}\right)$ are uniformly ultimately bounded, and the ultimate bound is given by

$$
\left\{\left\|e_{R}\right\|^{2}+\left\|e_{\Omega}\right\|^{2} \leq \frac{\lambda_{M}\left(M_{22}\right)}{\lambda_{m}\left(M_{21}\right) \lambda_{m}\left(W_{2}\right)} \varepsilon_{R}\right\} .
$$

An estimate of the region of attraction is given by the region where the following inequality is satisfied: $\frac{1}{2} e_{\Omega}(0) \cdot J e_{\Omega}(0)$ $+k_{R} \Psi\left(R(0), R_{d}(0)\right)+c_{2} e_{R}(0) \cdot e_{\Omega}(0)<\lambda_{m}\left(M_{21}\right) \psi_{2}\left(2-\psi_{2}\right)$.

Proof. See Appendix 1.2.

From (16), the initial attitude error should be less than $180^{\circ}$, in terms of the rotation angle about the eigenaxis between $R$ and $R_{d}$. We can further show that the attitude tracking errors exponentially converges to (19), where the size of the ultimate bound can be reduced by the controller parameter $\varepsilon_{R}$. The ultimate bound is expressed in terms of the attitude error vector $e_{R}$, which becomes zero when the attitude error is $180^{\circ}$ as well. But, these undesired attitudes are guaranteed to be avoided for the initial conditions in the given estimate of the region of attraction. All of these results can be applied to a nonlinear robust control problem for the attitude dynamics of any rigid body.

Asymptotic tracking of the quadrotor attitude does not require specification of the thrust magnitude. As an auxiliary problem, the thrust magnitude can be chosen in many different ways to achieve an additional translational motion objective. For example, it can be used to asymptotically track a quadrotor altitude command [18].

Since the translational motion of the quadrotor UAV can only be partially controlled; this flight mode is most suitable for short time periods where an attitude maneuver is to be completed.

\section{POSITION CONTROLLED FLIGHT MODE}

We now introduce a nonlinear controller for the position controlled flight mode. This flight mode requires analysis of the coupled translational and rotational equations of motion; hence, we make use of the notation and analysis in the prior section to describe the properties of the closed loop system in this flight mode.

\subsection{Position tracking errors}

An arbitrary smooth position tracking command $x_{d}(t) \in \mathbb{R}^{3}$ is chosen. The position tracking errors for the position and the velocity are given by:

$$
\begin{aligned}
& e_{x}=x-x_{d}, \\
& e_{v}=v-\dot{x}_{d} .
\end{aligned}
$$

Following the prior definition of the attitude error and the angular velocity error, we define

$$
e_{R}=\frac{1}{2}\left(R_{c}^{T} R-R^{T} R_{c}\right)^{\vee}, \quad e_{\Omega}=\Omega-R^{T} R_{c} \Omega_{c},
$$

and the computed attitude $R_{c}(t) \in \mathrm{SO}(3)$ and computed angular velocity $\Omega_{c} \in \mathbb{R}^{3}$ are given by

$$
R_{c}=\left[b_{1_{c}} ; b_{3_{c}} \times b_{1_{c}} ; b_{3_{c}}\right], \quad \hat{\Omega}_{c}=R_{c}^{T} \dot{R}_{c},
$$

where $b_{3_{c}} \in \mathrm{S}^{2}$ is defined by

$$
b_{3_{c}}=-\frac{-k_{x} e_{x}-k_{v} e_{v}-m g e_{3}+m \ddot{x}_{d}+\mu_{x}}{\left\|-k_{x} e_{x}-k_{v} e_{v}-m g e_{3}+m \ddot{x}_{d}+\mu_{x}\right\|},
$$

and $b_{1 c} \in \mathrm{S}^{2}$ is selected to be orthogonal to $b_{3 c}$, thereby guaranteeing that $R_{c} \in \mathrm{SO}(3)$. The constants $k_{x}, k_{v}$ are positive, and the control input term $\mu_{x}$ is defined later in (29). We assume that

$$
\left\|-k_{x} e_{x}-k_{v} e_{v}-m g e_{3}+m \ddot{x}_{d}+\mu_{x}\right\| \neq 0,
$$

and the commanded acceleration is uniformly bounded such that

$$
\left\|-m g e_{3}+m \ddot{x}_{d}\right\|<B
$$

for a given positive constant $B$.

\subsection{Position tracking controller}

The nonlinear controller for the position controlled flight mode, described by control expressions for the thrust magnitude and the moment vector, is:

$$
\begin{aligned}
& f=\left(k_{x} e_{x}+k_{v} e_{v}+m g e_{3}-m \ddot{x}_{d}-\mu_{x}\right) \cdot R e_{3}, \\
& M=-k_{R} e_{R}-k_{\Omega} e_{\Omega}+\Omega \times J \Omega \\
& \text { - } J\left(\hat{\Omega} R^{T} R_{c} \Omega_{c}-R^{T} R_{c} \dot{\Omega}_{c}+\mu_{R}\right) \text {, } \\
& \mu_{x}=-\frac{\delta_{x}^{\tau+2} e_{B}\left\|e_{B}\right\|^{\tau}}{\delta_{x}^{\tau+1}\left\|e_{B}\right\|^{\tau+1}+\varepsilon_{x}^{\tau+1}}, \\
& e_{B}=e_{v}+\frac{c_{1}}{m} e_{x}, \\
& \mu_{R}=-\frac{\delta_{R}^{2} e_{A}}{\delta_{R}\left\|e_{A}\right\|+\varepsilon_{R}},
\end{aligned}
$$




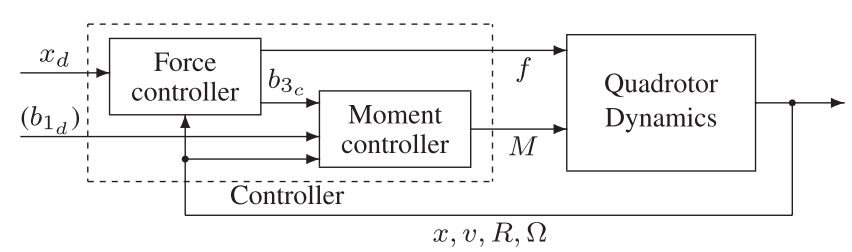

Fig. 2. Controller structure for position controlled flight mode.

$$
e_{A}=e_{\Omega}+c_{2} J^{-1} e_{R}
$$

where $k_{x}, k_{v}, k_{R}, k_{\Omega}, c_{1}, c_{2}, \varepsilon_{x}, \varepsilon_{R}, \tau$ are positive constants, and $\tau>2$.

The nonlinear controller given by (27), (28) can be given a backstepping interpretation. The computed attitude $R_{c}$ given in (23) is selected so that the thrust axis $-b_{3}$ of the quadrotor UAV tracks the computed direction given by $-b_{3_{c}}$ in (24), which is a direction of the thrust vector that achieves position tracking. The moment expression (28) causes the attitude of the quadrotor UAV to asymptotically track $R_{c}$ and the thrust magnitude expression (27) achieves asymptotic position tracking.

The closed loop system for this position controlled flight mode is illustrated in Fig. 2. The corresponding closed loop control system is described by (2)-(5), using the controller expressions (27)-(32). We now state the result that the tracking errors $\left(e_{x}, e_{v}, e_{R}, e_{\Omega}\right)$ are uniformly ultimately bounded.

Proposition 3. (Robustness of Position Controlled Flight Mode) Suppose that the initial conditions satisfy

$$
\begin{aligned}
& \Psi\left(R(0), R_{c}(0)\right)<\psi_{1}<1, \\
& \left\|e_{x}(0)\right\|<e_{x_{\max }},
\end{aligned}
$$

for positive constants $\psi_{1}, e_{x_{\max }}$. Consider the control inputs $f$, $M$ defined in (27)-(32). For positive constants $k_{x}, k_{v}$, we choose positive constants $c_{1}, c_{2}, k_{R}, k_{\Omega}, \varepsilon_{x}, \varepsilon_{R}$ such that

$$
\begin{aligned}
& c_{1}<\min \left\{\frac{4 m k_{x} k_{v}(1-\alpha)^{2}}{k_{v}^{2}(1+\alpha)^{2}+4 m k_{x}(1-\alpha)}, \sqrt{k_{x} m}\right\}, \\
& c_{2}<\min \left\{\frac{4 k_{\Omega} k_{R} \lambda_{m}(J)^{2}}{k_{\Omega}^{2} \lambda_{M}(J)+4 k_{R} \lambda_{m}(J)^{2}}, \sqrt{k_{R} \lambda_{m}(J)}\right\}, \\
& \lambda_{m}\left(W_{2}\right)>\frac{\left\|W_{12}\right\|^{2}}{4 \lambda_{m}\left(W_{1}\right)}, \\
& \left.\varepsilon_{x}+\varepsilon_{R}<\frac{\min \left\{\lambda_{m}\left(M_{11}\right), \lambda_{m}\left(M_{21}\right)\right\}}{\min \left\{e_{x_{\max }}^{2}, \psi_{1}\left(2-\psi_{1}\right)\right\}} \lambda_{M}\left(M_{12}\right), \lambda_{M}\left(M_{22}^{\prime}\right)\right\}
\end{aligned}
$$

where $\alpha=\sqrt{\psi_{1}\left(2-\psi_{1}\right)}$, and the matrices $M_{11}, M_{12}, M_{21}, M_{22}^{\prime}$, $W_{1}, W_{12}, W_{2}, W \in \mathbb{R}^{2 \times 2}$ are given by

$$
\begin{aligned}
& M_{11}=\frac{1}{2}\left[\begin{array}{cc}
k_{x} & -c_{1} \\
-c_{1} & m
\end{array}\right], \quad M_{12}=\frac{1}{2}\left[\begin{array}{cc}
k_{x} & c_{1} \\
c_{1} & m
\end{array}\right] \text {, } \\
& W_{1}=\left[\begin{array}{cc}
\frac{c_{1} k_{x}}{m}(1-\alpha) & -\frac{c_{1} k_{v}}{2 m}(1+\alpha) \\
-\frac{c_{1} k_{v}}{2 m}(1+\alpha) & k_{v}(1-\alpha)-c_{1}
\end{array}\right], \\
& W_{12}=\left[\begin{array}{cc}
\frac{c_{1}}{m}\left(B+\delta_{x}\right) & 0 \\
B+\delta_{x}+k_{x} e_{x_{\max }} & 0
\end{array}\right], \\
& W_{2}=\left[\begin{array}{cc}
\frac{c_{2} k_{R}}{\lambda_{M}(J)} & -\frac{c_{2} k_{\Omega}}{2 \lambda_{m}(J)} \\
-\frac{c_{2} k_{\Omega}}{2 \lambda_{m}(J)} & k_{\Omega}-c_{2}
\end{array}\right], \\
& W=\left[\begin{array}{cc}
\lambda_{m}\left(W_{1}\right) & -\frac{1}{2}\left\|W_{12}\right\|_{2} \\
-\frac{1}{2}\left\|W_{12}\right\|_{2} & \lambda_{m}\left(W_{2}\right)
\end{array}\right] .
\end{aligned}
$$$$
M_{21}=\frac{1}{2}\left[\begin{array}{cc}
k_{R} & -c_{2} \\
-c_{2} & \lambda_{m}(J)
\end{array}\right], \quad M_{22}^{\prime}=\frac{1}{2}\left[\begin{array}{cc}
\frac{2 k_{R}}{2-\psi_{1}} & c_{2} \\
c_{2} & \lambda_{M}(J)
\end{array}\right],
$$

Then, the tracking errors $\left(e_{x}, e_{v}, e_{R}, e_{\Omega}\right)$ are uniformly ultimately bounded, and the ultimate bound is given by

$$
\begin{aligned}
& \left\{\left\|e_{x}\right\|^{2}+\left\|e_{v}\right\|^{2}+\left\|e_{R}\right\|^{2}+\left\|e_{\Omega}\right\|^{2}\right. \\
& \left.\quad<\frac{\max \left\{\lambda_{M}\left(M_{12}\right), \lambda_{M}\left(M_{22}^{\prime}\right)\right\}}{\min \left\{\lambda_{m}\left(M_{11}\right), \lambda_{m}\left(M_{21}\right)\right\} \lambda_{m}(W)}\left(\varepsilon_{x}+\varepsilon_{R}\right)\right\} .
\end{aligned}
$$

An estimate of the region of attraction is given by the region where the following inequality is satisfied: $\frac{1}{2} k_{x}\left\|e_{x}(0)\right\|^{2}+\frac{1}{2} m\left\|e_{v}(0)\right\|^{2}+c_{1} e_{x}(0) \cdot e_{v}(0)+\frac{1}{2} e_{\Omega}(0) \cdot J e_{\Omega}(0)+$ $k_{R} \Psi\left(R(0), R_{d}(0)\right)+c_{2} e_{R}(0) \cdot e_{\Omega}(0)<\min \left\{\lambda_{m}\left(M_{11}\right), \lambda_{m}\left(M_{21}\right)\right\}$ $\min \left\{e_{x_{\max }}^{2}, \psi_{1}\left(2-\psi_{1}\right)\right\}$.

Proof. See Appendix 1.3.

This proposition shows that the proposed control system is robust to bounded, unstructured uncertainties in the dynamics of a quadrotor UAV. Similar to Proposition 2, the ultimate bound can be arbitrarily reduced by choosing smaller $\varepsilon_{x}, \varepsilon_{R}$, and it is possible to obtain exponential attractiveness. 


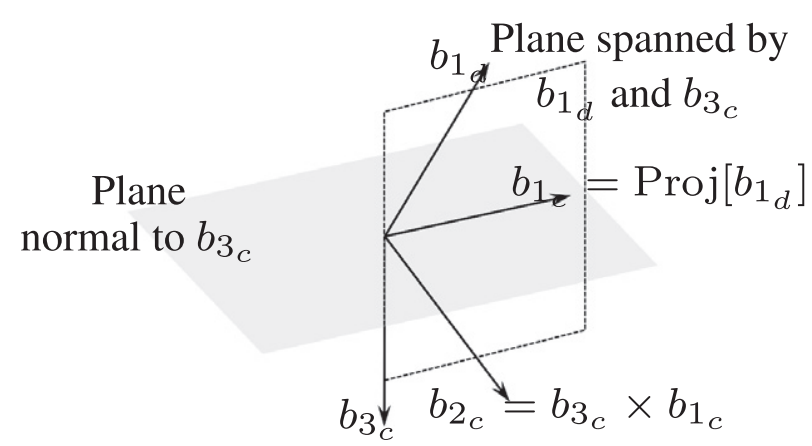

Fig. 3. Convergence property of the first body-fixed axis: in the proposed control system, $b_{3_{c}}$ is determined by (24). We choose a desired direction of the first body fixed axis, namely $b_{1_{d}}$ that is not parallel to $b_{3_{c}}$, and project it on to the plane normal to $b_{3_{c}}$ to obtain $b_{1_{c}}$. This guarantees that the first body-fixed axis converges to $b_{1_{c}}$, and therefore it asymptotically lies in the plane spanned by $b_{1_{d}}$ and $b_{3_{c}}$. As $b_{3 c}$ converges to the direction of $g e_{3}-\ddot{x}_{d}$ in (24), this allows us to specify the direction of the first body-fixed axis in the plane normal to $g e_{3}-\ddot{x}_{d}$. For all cases, the ultimate convergence error is described by (39).

Proposition 3 requires that the initial attitude error is less than $90^{\circ}$ in (33). Suppose that this is not satisfied, i.e. $1 \leq \Psi\left(R(0), R_{c}(0)\right)<2$. We can still apply Proposition 2 , which states that the attitude error exponentially decreases until it enters the ultimate bound given by (19). If the constant $\varepsilon_{R}$ is sufficiently small, we can guarantee that the attitude error function decreases to satisfy (33) in a finite time. Therefore, by combining the results of Propositions 2 and 3, we can show ultimate boundedness of the tracking errors when $\Psi\left(R(0), R_{c}(0)\right)<2$.

Proposition 4. (Robustness of Position Controlled Flight Mode with a Larger Initial Attitude Error) Suppose that the initial conditions satisfy

$$
1 \leq \Psi\left(R(0), R_{c}(0)\right)<\psi_{2}<2,
$$

$$
\left\|e_{x}(0)\right\|<e_{x_{\max }},
$$

for a constant $\psi_{2}, e_{x_{\max }}$. Consider the control inputs $f$, $M$ defined in (27)-(32), where the control parameters $k_{x}, k_{v}, k_{R}, k_{\Omega}, c_{1}, c_{2}, \varepsilon_{x}, \varepsilon_{R}$ satisfy (35)-(38) for a positive constant $\psi_{1}<1$. If the constant $\varepsilon_{R}$ is sufficiently small such that

$$
\varepsilon_{R}<\frac{\lambda_{m}\left(M_{21}\right) \lambda_{m}\left(W_{2}\right)}{\lambda_{M}\left(M_{22}\right)} \psi_{1}\left(2-\psi_{1}\right)
$$

then the tracking errors $\left(e_{x}, e_{v}, e_{R}, e_{\Omega}\right)$ are uniformly ultimately bounded, and the ultimate bound is given by (39).

Proof. See Appendix 1.4.

\subsection{Direction of the first body-fixed axis}

As described above, the construction of the orthogonal matrix $R_{c}$ involves having its third column $b_{3_{c}}$ specified by a normalized feedback function, and its first column $b_{1_{c}}$ is chosen to be orthogonal to the third column. The unit vector $b_{1_{c}}$ can be arbitrarily chosen in the plane normal to $b_{3_{c}}$, which corresponds to a one-dimensional degree of choice. This reflects the fact that the quadrotor UAV has four control inputs that are used to track a three-dimensional position command.

By choosing $b_{1 c}$ properly, we constrain the asymptotic direction of the first body-fixed axis. Here, we propose to specify the projection of the first body-fixed axis onto the plane normal to $b_{3_{c}}$. In particular, we choose a desired direction $b_{1_{d}} \in \mathrm{S}^{2}$, that is not parallel to $b_{3_{c}}$, and $b_{1_{c}}$ is selected as $b_{1_{c}}=\operatorname{Proj}\left[b_{1_{d}}\right]$, where Proj[·] denotes the normalized projection onto the plane perpendicular to $b_{3 c}$ (see Figure 3). In this case, the first body-fixed axis does not converge to $b_{1_{d}}$, but it converges to the projection of $b_{1_{d}}$, i.e. $b_{1} \rightarrow b_{1_{c}}=\operatorname{Proj}\left[b_{1_{d}}\right]$ as $t \rightarrow \infty$, up to the ultimate bound described by (39). In other words, the first body-fixed axis converges to a small neighborhood of the intersection of the plane normal to $b_{3_{c}}$ and the plane spanned by $b_{3 c}$ and $b_{1 d}$. This can be used to specify the heading direction of a quadrotor UAV in the horizontal plane.

Proposition 5. (Specified Asymptotic Direction of First Body-Fixed Axis) Consider the moment vector $\mathrm{M}$ defined in (28) and the thrust magnitude $f$ defined in (27) satisfying the assumptions of Propositions 3 and 4.

In addition, the first column of $R_{c}$, namely $b_{1_{c}}$ is constructed as follows. We choose $b_{1_{d}}(t) \in \mathrm{S}^{2}$, and we assume that it is not parallel to $b_{3_{c}}$. The unit vector $b_{1_{c}}$ is constructed by projecting $b_{1_{d}}$ onto the plane normal to $b_{3_{c}}$, and normalizing it:

$$
b_{1_{c}}=-\frac{1}{\left\|b_{3_{c}} \times b_{1_{d}}\right\|}\left(b_{3_{c}} \times\left(b_{3_{c}} \times b_{1_{d}}\right)\right) \text {. }
$$

Then, the conclusions of Propositions 3 and 4 hold, and the first body-fixed axis asymptotically lies in the plane spanned by $b_{1 d}$ and $g e_{3}-\ddot{x}_{d}$.

In the special case where $\ddot{x}_{d}=0$, we can choose $b_{1 d}$ in the horizontal plane. Then, the first body-fixed axis asymptotically converges to $b_{1 d}$.

These additional properties of the closed loop can be interpreted as characterizing the asymptotic direction of the first body-fixed axis and the asymptotic direction of the third body-fixed axis as it depends on the commanded vehicle acceleration. These physical properties may be of importance in some flight maneuvers. 


\subsection{Velocity controlled flight mode}

Suppose that an arbitrary velocity tracking command $t \rightarrow v_{d} \in \mathbb{R}^{3}$ is given. The velocity tracking error is given by:

$$
e_{v}=v-v_{d}
$$

It is straightforward to construct a velocity tracking controller, by using the results of the prescribed position tracking controller. More explicitly, the controller structure of a velocity controlled flight mode can be considered as a special case of (27)-(32), where $c_{1}=k_{x}=0$ and $\ddot{x}_{d}=\dot{v}_{d}$. We can show the ultimate boundedness of the tracking errors similar to Propositions 3 and 4 . A similar procedure to construct a velocity tracking controller from a position tracking controller is available in [18].

\section{NUMERICAL EXAMPLES}

Numerical results are presented to demonstrate the applicability of the proposed approach for performing complex flight maneuvers. The parameters are chosen to match a quadrotor UAV described in [31].

$$
\begin{aligned}
& J=[0.0820,0.0845,0.1377] \mathrm{kg}-\mathrm{m}^{2}, m=4.34 \mathrm{~kg} \\
& d=0.315 \mathrm{~m}, \quad c_{\tau f}=8.004 \times 10^{-3} \mathrm{~m} .
\end{aligned}
$$

The controller parameters are chosen as follows:

$$
\begin{aligned}
& k_{x}=59.02, \quad k_{v}=24.30, \quad k_{R}=8.81, \quad k_{\Omega}=1.54 \\
& c_{1}=3.6, \quad c_{2}=0.6, \quad \varepsilon_{x}=\varepsilon_{R}=0.04 .
\end{aligned}
$$

We consider a fixed disturbance for the translational dynamics, and an oscillatory disturbance for the rotational dynamics as follows:

$$
\begin{aligned}
& \Delta_{x}=[2.50,1.25,2.00]^{T} \mathrm{~N}, \\
& \Delta_{R}(t)=\frac{1}{\sqrt{3}}[\sin (8 \pi t), \sin (\pi t), \cos (4 \pi t)]^{T} \mathrm{Nm} .
\end{aligned}
$$

The corresponding bounds of the disturbances are given by $\delta_{x}=4.34$ and $\delta_{R}=1$. We assume that the thrust of each rotor is bounded by $\left|f_{i}\right|<30 \mathrm{~N}$ for $i \in\{1,2,3,4\}$. When computing the control inputs, we consider the following measurement errors. Unbiased measurement noise that is uniformly distributed between -0.05 and $0.05 \mathrm{~m}$ is added to each element of the position variable $x$. Biased measurement noise that is uniformly distributed between $[-0.4,0,-0.25]^{T}$ and $[-0.2,0.2,-0.025]^{T} \mathrm{rad} / \mathrm{s}$ is added to the angular velocity vector $\Omega$. We consider the following two cases.
Case I: Position Controlled Flight Modes. The initial conditions are given by

$$
\begin{aligned}
& x(0)=[0.1,0,0]^{T} \mathrm{~m}, \quad v(0)=[0,0,0]^{T} \mathrm{~m} / \mathrm{s}, \\
& R(0)=\exp \left(0.99 \pi \hat{e}_{1}\right), \quad \Omega(0)=[0,0,0]^{T} \mathrm{rad} / \mathrm{s},
\end{aligned}
$$

where $e_{1}=[1,0,0] \in \mathbb{R}^{3}$. The desired position command is given by

$$
x_{d}(t)=[0,0,0]^{T} \mathrm{~m},
$$

and the desired heading direction is fixed as $\vec{b}_{1_{d}}=[1,0,0]^{T}$. This describes the case of a quadrotor UAV recovering from an initially upside-down configuration.

The initial attitude error is given by $1 \leq(\Psi(0)=$ $1.9995<2$, and therefore, it corresponds to Proposition 4 that is based on both the attitude controlled flight mode and the position controlled flight mode.

Fig. 4 illustrates the excellent convergence properties of the proposed control system for a large initial attitude error, where the terminal position tracking error is $1.2 \mathrm{~cm}$. Fig. 5 shows the relatively poor tracking performance and the slower convergence rate of a controller without the robust control input terms proposed in this paper.

Case II: Transition Between Several Flight Modes. This flight maneuver consists of a sequence of five flight modes, including a rotation by $720^{\circ}$ (see Fig. 6).

(a) Velocity controlled flight mode $(t \in[0,4))$

$$
\begin{aligned}
& v_{d}(t)=[1+0.5 t, 0.2 \sin (2 \pi t),-0.1], \\
& b_{1_{d}}(t)=[1,0,0] .
\end{aligned}
$$

(b) Attitude controlled flight mode $(t \in[4,6))$ : rotation about the second body-fixed axis by $720^{\circ}$

$$
R_{d}(t)=\exp \left(2 \pi(t-4) \hat{e}_{2}\right) .
$$

(c) Position controlled flight mode $(t \in[6,10))$

$$
x_{d}(t)=\left[11-\frac{t}{2}, 0,0\right], \quad b_{1 d}(t)=[1,0,0] .
$$

(d) Attitude controlled flight mode $(t \in[10,11))$ : rotation about the first body-fixed axis by $360^{\circ}$

$$
R_{d}(t)=\exp \left(2 \pi(t-10) \hat{e}_{1}\right) .
$$

(e) Position controlled flight mode $(t \in[11,15])$

$$
x_{d}(t)=\left[\frac{75}{4}-\frac{5}{4} t, 0,0\right], \quad b_{1_{d}}(t)=[1,0,0] .
$$

Initial conditions are same as the first case. 


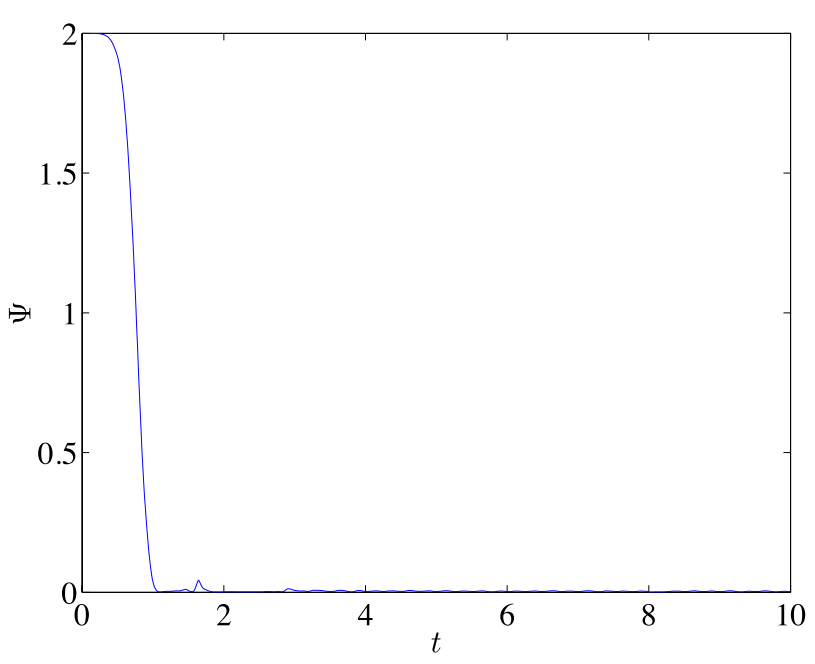

(a) Attitude error function $\Psi$
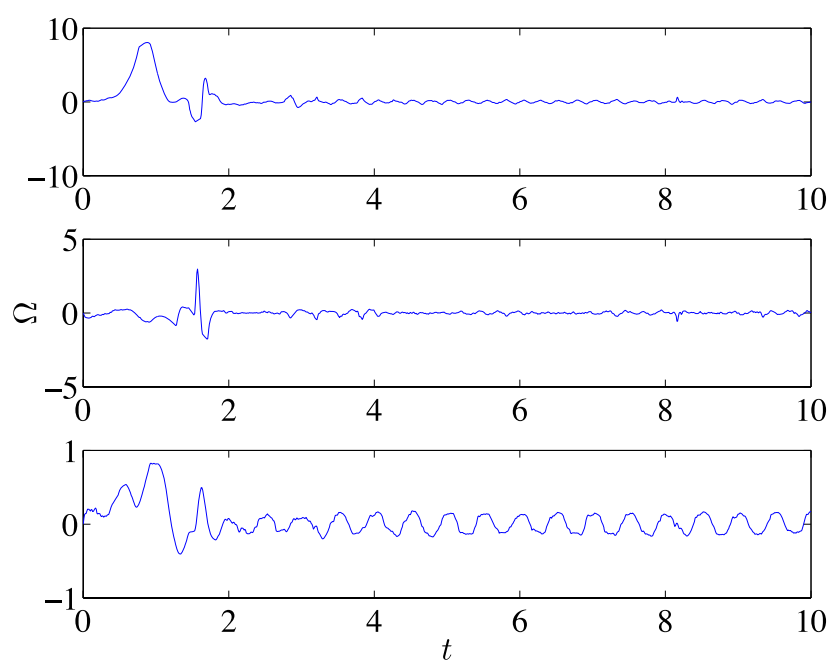

(c) Angular velocity error $e_{\Omega}(\mathrm{rad} / \mathrm{sec})$
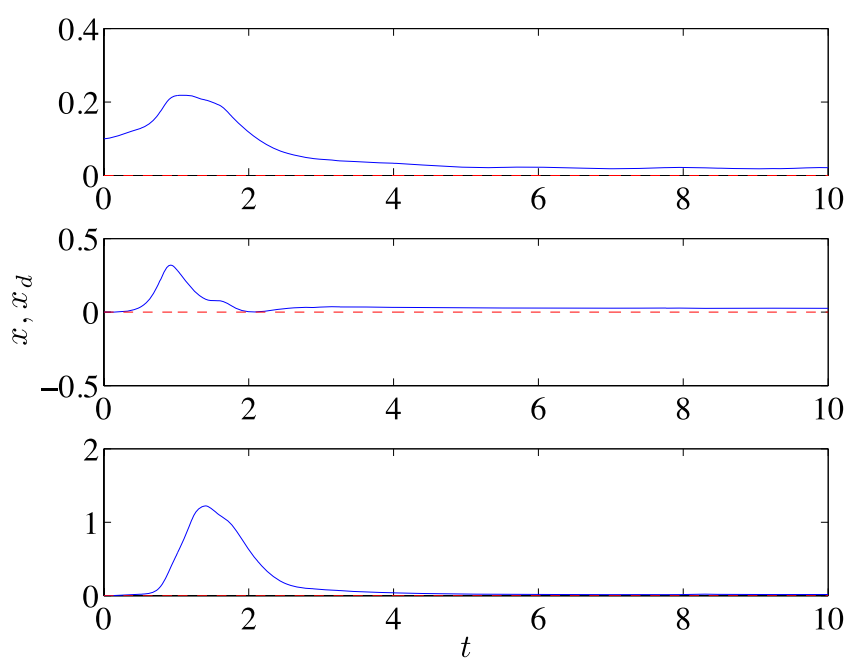

(b) Position error $e_{x}(\mathrm{~m})$
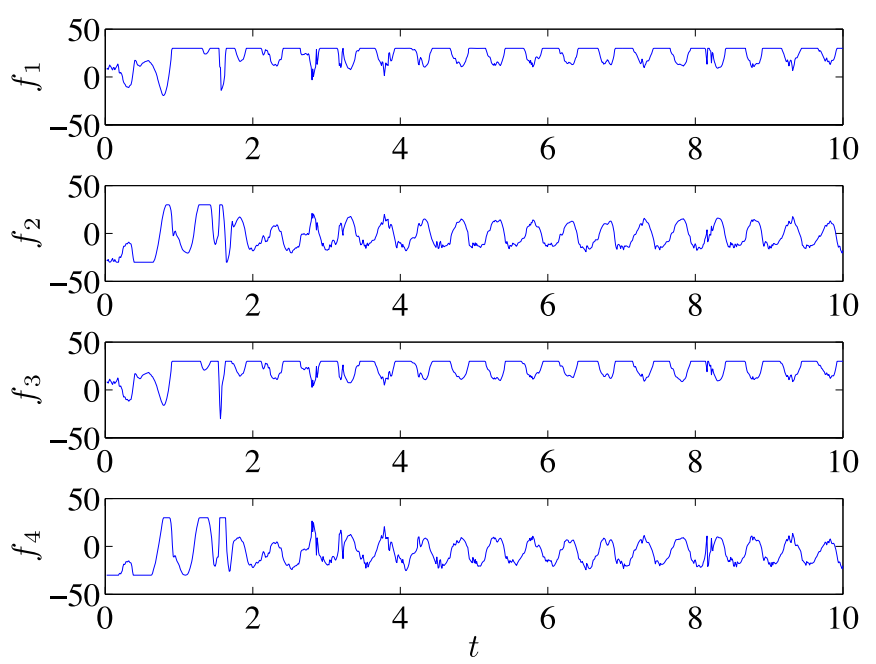

(d) Thrust of each rotor $(\mathrm{N})$

Fig. 4. Case II: robust position controlled flight mode to recover from an initially upside-down configuration.

The second case involves transitions between several flight modes. It begins with a velocity controlled flight mode. As the initial attitude error function is less than 1 , the velocity tracking error exponentially converges as shown at Fig. 7d, and the first body-fixed axis asymptotically lies in the plane spanned by $b_{1_{d}}=e_{1}$ and $g e_{3}-\dot{v}_{d}$. Since $\left\|\dot{v}_{d}\right\| \ll g$, the first body-fixed axis remains close to the plane spanned by $e_{1}$ and $e_{3}$, as illustrated in Fig. 7e.

This is followed by an attitude tracking mode to rotate the quadrotor by $720^{\circ}$ about the second body-fixed axis according to Proposition 2. As discussed in Section IV, the thrust magnitude $f$ can be arbitrarily chosen in an attitude controlled flight mode. Here, we choose the thrust magnitude given by

$$
f(t)=\left(k_{x}\left(x(t)-x_{c}\right)+k_{v} v(t)+m g e_{3}\right) \cdot R(t) e_{3},
$$

which is equivalent to the thrust magnitude for the position controlled flight mode given in (27), when $x_{d}(t)=x_{c}=[8,0$, $0]$. This does not guarantee asymptotic convergence of the quadrotor UAV position to $[8,0,0]$ since the direction of the total thrust is determined by the given attitude command. But, it has the effect that the position of the quadrotor UAV stays close to $x_{c}$, as illustrated at Fig. $7 \mathrm{~b}$.

Next, a position tracking mode is again engaged, and the quadrotor UAV soon follows a straight line. Another attitude tracking mode and position tracking mode are engaged to rotate the quadrotor by $360^{\circ}$ about the direction of the second body-fixed axis. The thrust magnitude is chosen as 


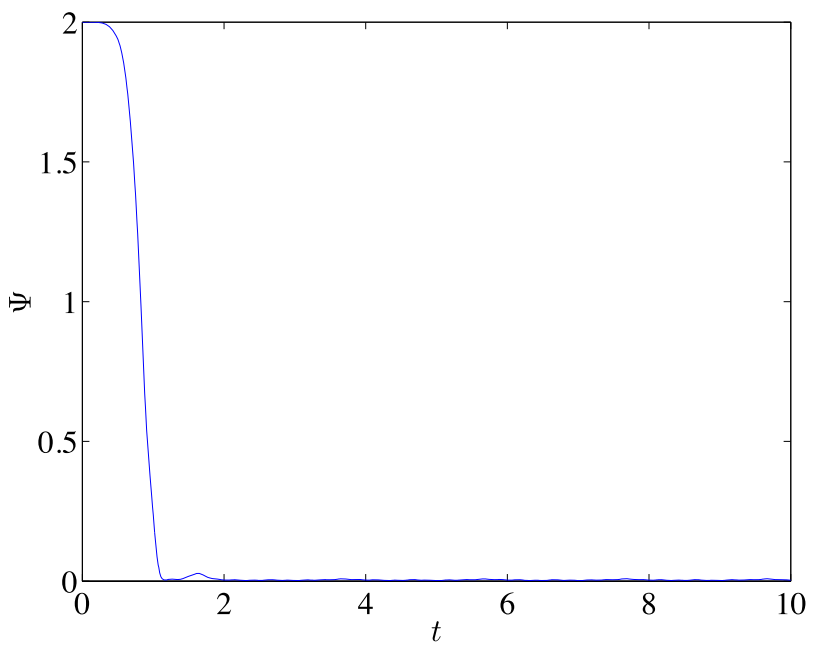

(a) Attitude error function $\Psi$
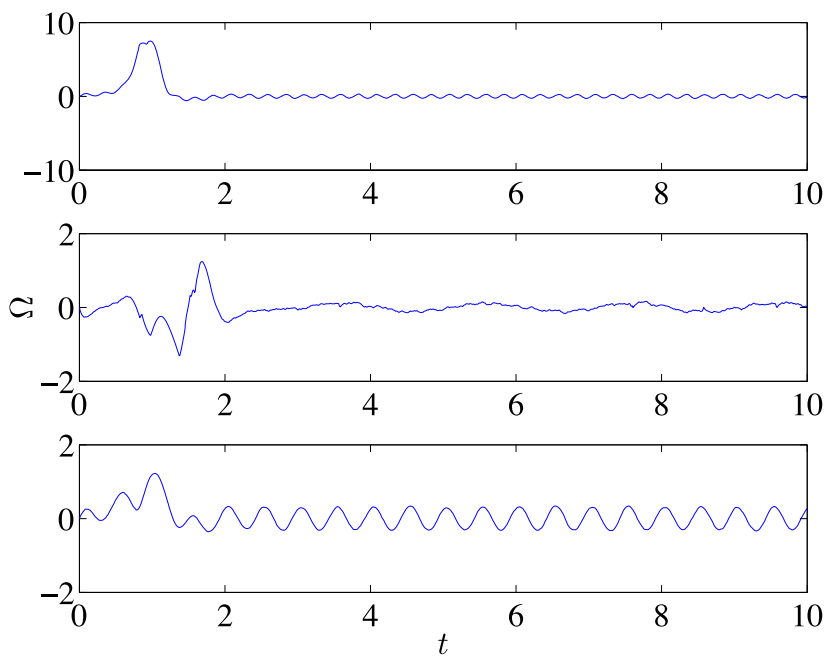

(c) Angular velocity error $e_{\Omega}(\mathrm{rad} / \mathrm{sec})$
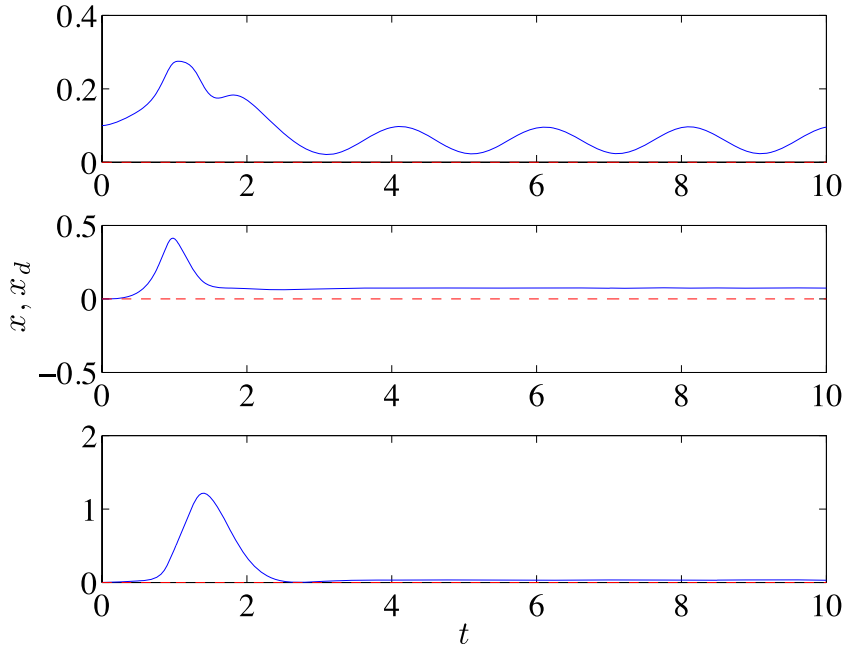

(b) Position error $e_{x}(\mathrm{~m})$
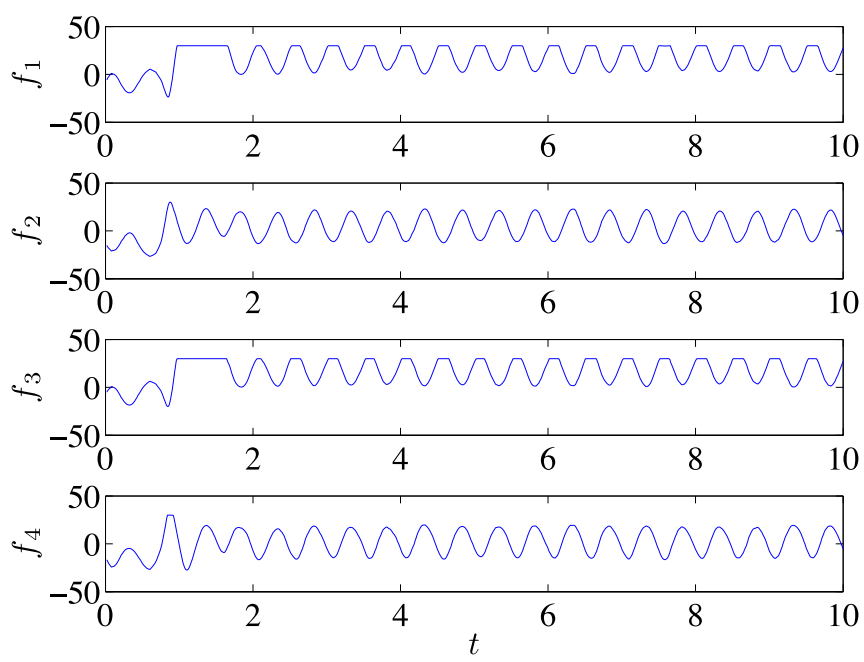

(d) Thrust of each rotor $(\mathrm{N})$

Fig. 5. Case II: robust position controlled flight mode to recover from an initially upside-down configuration. The robust control input terms are set to zero, i.e. $\mu_{x}=\mu_{R}=0$, for comparison with Fig. 4 .

(45), where $x_{c}=[6,0,0]$, to make the position of the quadrotor UAV remain close to $x_{c}$ during this attitude maneuver, as discussed above. For the position tracking modes (c) and (e), we have $\ddot{x}_{d}=0$, and $b_{1_{d}}$ lies in the horizontal plane. Therefore, according to Proposition 5, the first body-fixed $b_{1}$ asymptotically converges to $b_{1_{d}}$, as shown at Figure 7e. These illustrate that by switching between an attitude mode and a position and heading flight mode, the quadrotor UAV can perform the prescribed complex aerobatic maneuver. Figure 8 shows the relatively poor tracking performance and slower convergence rate in the absence of the robust control input terms proposed in this paper.

\section{CONCLUSIONS}

We presented a global dynamic model for a quadrotor $\mathrm{UAV}$, and we developed tracking controllers that are robust to unstructured uncertainties for three different flight modes; these were developed in terms of the special Euclidean group and are intrinsic and coordinate-free, thereby avoiding the singularities of Euler angles and the ambiguities of quaternions in representing attitude. The proposed geometric based controllers for the three flight modes have tracking errors that are uniformly ultimately bounded. By switching between different controllers for these flight modes, we have 


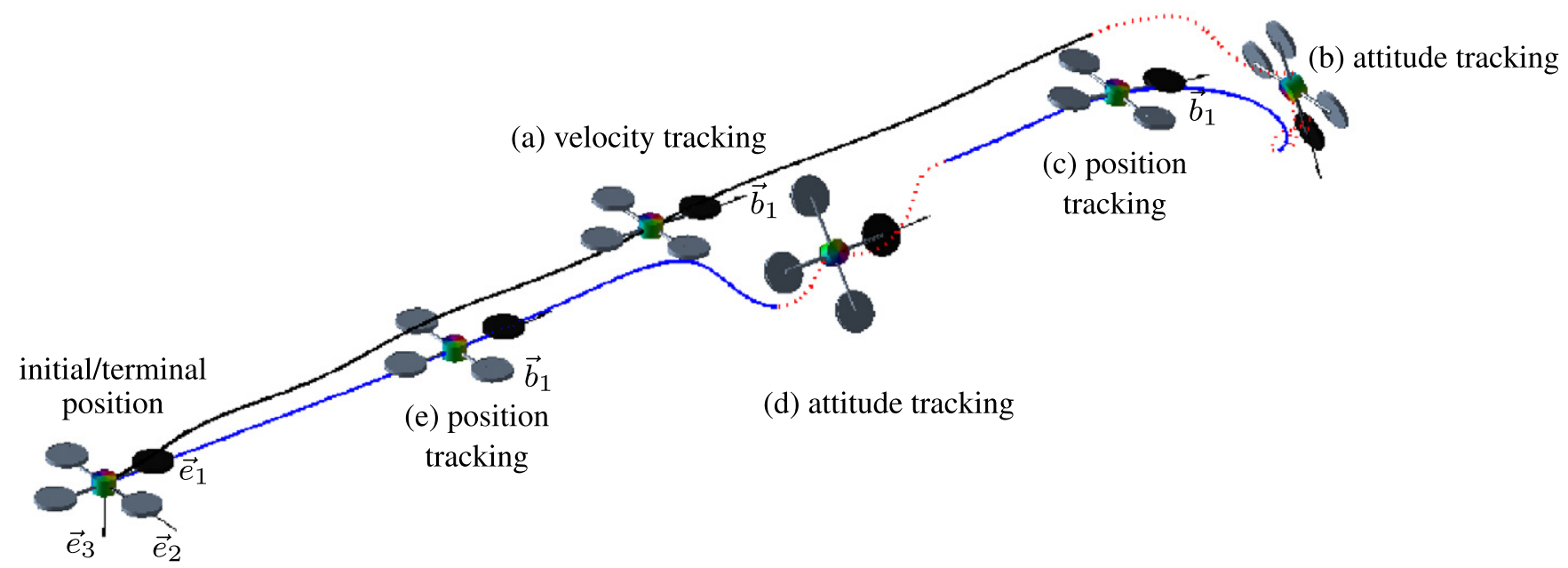

Fig. 6. Case II: complex maneuver of a quadrotor UAV involving a rotation by $720^{\circ}$ about $\vec{e}_{2}$ (b), and a rotation by $360^{\circ}$ about $\vec{e}_{1}(\mathrm{~d}$ ), with transitions between several flight modes. The direction of the first body-fixed axis is specified for velocity/position tracking modes ((a),(c),(e)).

demonstrated that the quadrotor UAV can perform complex aerobatic maneuvers. Several different complex flight maneuvers were demonstrated in the numerical examples.

But, this result is restricted to bounded and additive uncertainties in the quadrotor dynamics. Generalizations of the proposed control systems to measurement errors or control input saturations, and the corresponding experimental validations are deferred to future research.

\section{APPENDIX: PROPERTIES AND PROOFS}

\subsection{PROPERTIES OF THE HAT MAP}

The hat map $\uparrow: \mathbb{R}^{3} \rightarrow \mathfrak{s o}(3)$ is defined as

$$
\hat{x}=\left[\begin{array}{ccc}
0 & -x_{3} & x_{2} \\
x_{3} & 0 & -x_{1} \\
-x_{2} & x_{1} & 0
\end{array}\right]
$$

for $x=\left[x_{1} ; x_{2} ; x_{3}\right] \in \mathbb{R}^{3}$. This identifies the Lie algebra $\mathfrak{s o}(3)$ with $\mathbb{R}^{3}$ using the vector cross product in $\mathbb{R}^{3}$. The inverse of the hat map is referred to as the vee map, $\mathrm{V}: \mathfrak{s o}(3) \rightarrow \mathbb{R}^{3}$. Several properties of the hat map are summarized as follows.

$$
\begin{aligned}
& \hat{x} y=x \times y=-y \times x=-\hat{y} x, \\
& -\frac{1}{2} \operatorname{tr}[\hat{x} \hat{y}]=x^{T} y, \\
& \operatorname{tr}[\hat{x} A]=\operatorname{tr}[A \hat{x}]=\frac{1}{2} \operatorname{tr}\left[\hat{x}\left(A-A^{T}\right)\right]=-x^{T}\left(A-A^{T}\right)^{\mathrm{v}}, \\
& \hat{x} A+A^{T} \hat{x}=\left(\left\{\operatorname{tr}[A] I_{3 \times 3}-A\right\} x\right)^{\wedge},
\end{aligned}
$$

$$
R \hat{x} R^{T}=(R x)^{\wedge},
$$

for any $x, y \in \mathbb{R}^{3}, A \in \mathbb{R}^{3 \times 3}$, and $R \in \mathrm{SO}(3)$.

\subsection{Proof of Proposition 2}

We first find the error dynamics for $e_{R}, e_{\Omega}$, and define a Lyapunov function. Then, we find conditions on control parameters to guarantee the boundedness of tracking errors.

Attitude error dynamics. The attitude error dynamics for $\Psi, e_{R}, e_{\Omega}$ are developed in [30], and they are summarized as follows:

$$
\begin{gathered}
\dot{\Psi}=e_{R} \cdot e_{\Omega}, \\
\dot{e}_{R}=E\left(R, R_{d}\right) e_{\Omega}, \\
\dot{e}_{\Omega}=J^{-1}\left(-\Omega \times J \Omega+u+\Delta_{R}\right)+\hat{\Omega} R^{T} R_{d} \Omega_{d}-R^{T} R_{d} \dot{\Omega}_{d},
\end{gathered}
$$

where the matrix $E\left(R, R_{d}\right) \in \mathbb{R}^{3 \times 3}$ is given by

$$
E\left(R, R_{d}\right)=\frac{1}{2}\left(\operatorname{tr}\left[R^{T} R_{d}\right] I-R^{T} R_{d}\right) .
$$

We can show that $\left\|\left(R, R_{d}\right)\right\| \leq 1$ to obtain

$$
\left\|\dot{e}_{R}\right\| \leq\left\|e_{\Omega}\right\| .
$$

Substituting the control moment (13) into (54),

$$
J \dot{e}_{\Omega}=-k_{R} e_{R}-k_{\Omega} e_{\Omega}+\Delta_{R}+\mu_{R} .
$$

In short, the attitude error dynamics are given by (52), (53), (57), and they satisfy (56). 


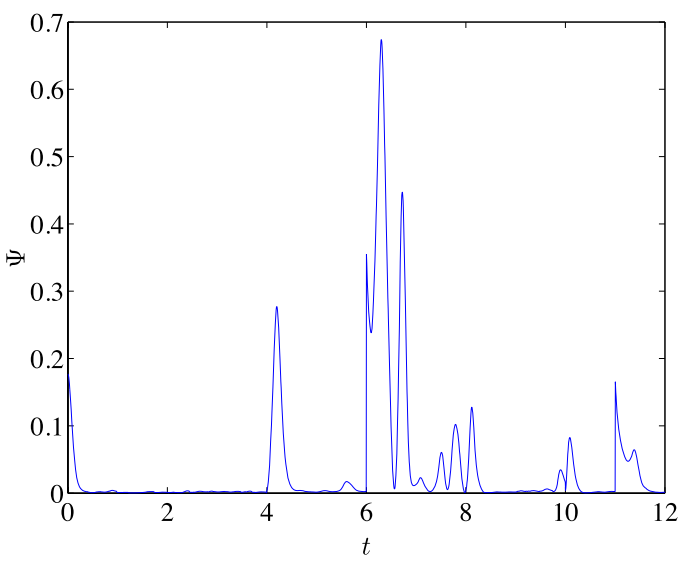

(a) Attitude error function $\Psi$
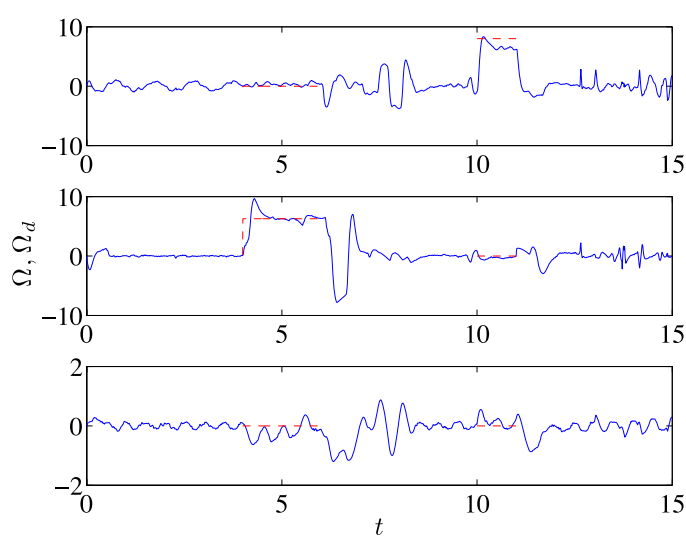

(c) Angular velocity ( $\Omega$ :solid, $\Omega_{d}$ :dotted, $\left.(\mathrm{rad} / \mathrm{sec})\right)$
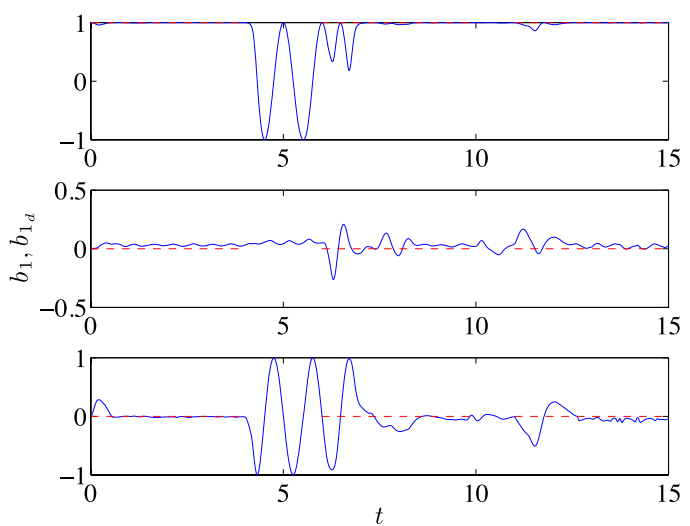

(e) First body-fixed axis $\left(b_{1}\right.$ :solid, $b_{1 d}$ :dotted)
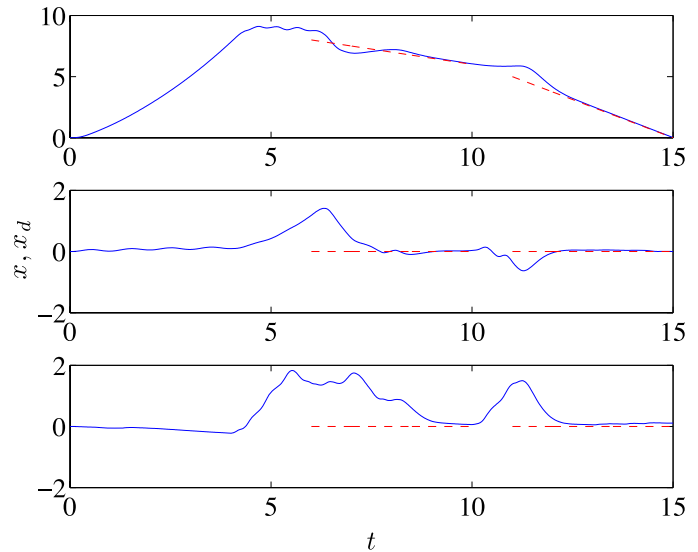

(b) Position ( $x$ :solid, $x_{d}$ :dotted, (m))
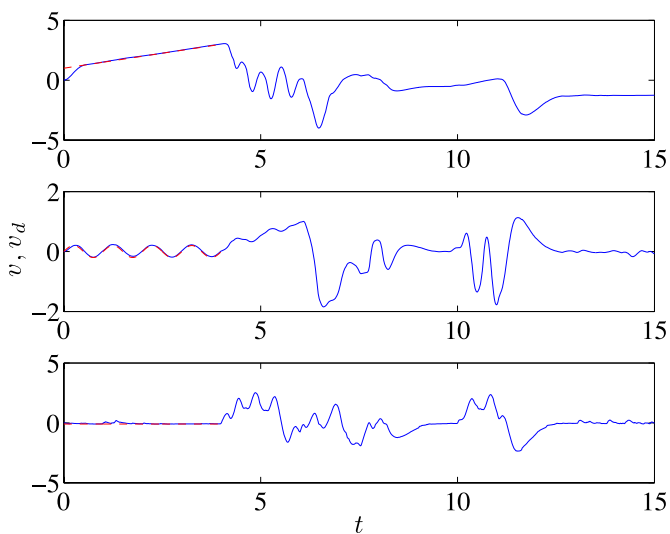

(d) Velocity ( $v$ :solid, $v_{d}$ :dotted, $(\mathrm{m} / \mathrm{s})$ )

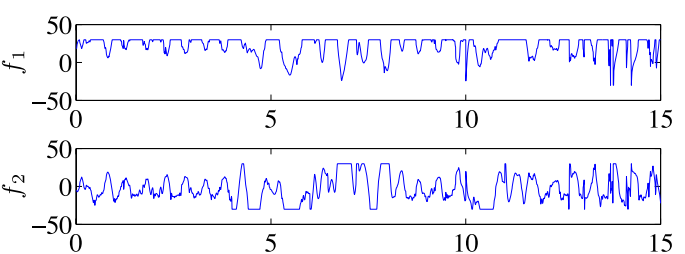

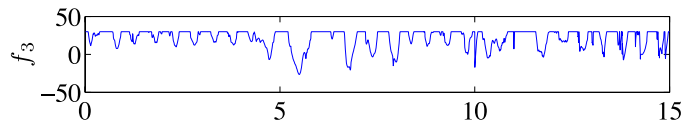

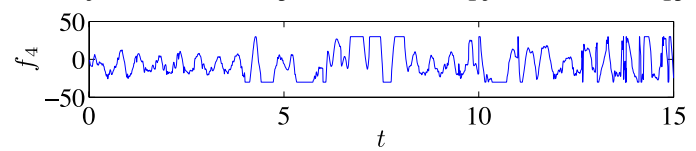

(f) Thrust of each rotor $(\mathrm{N})$

Fig. 7. Case II: transitions between several flight modes for a complex maneuver. 


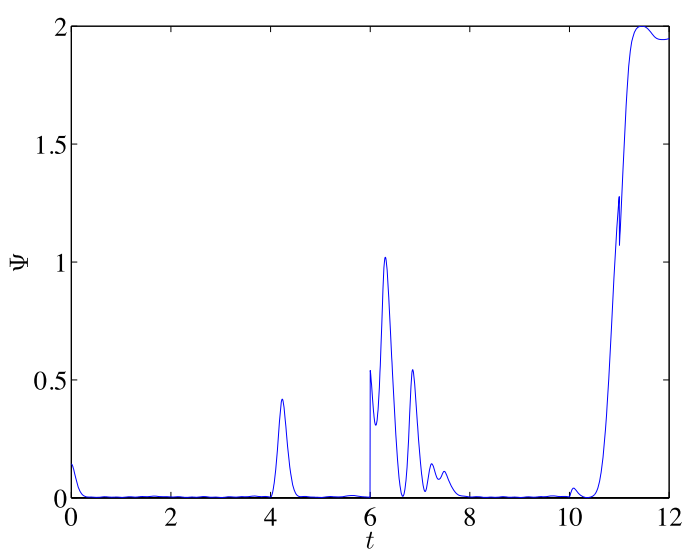

(a) Attitude error function $\Psi$
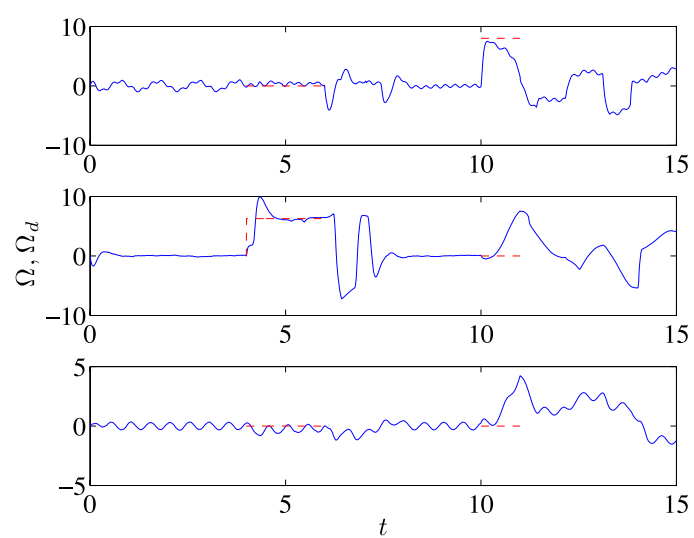

(c) Angular velocity ( $\Omega$ :solid, $\Omega_{d}$ :dotted, (rad/sec))
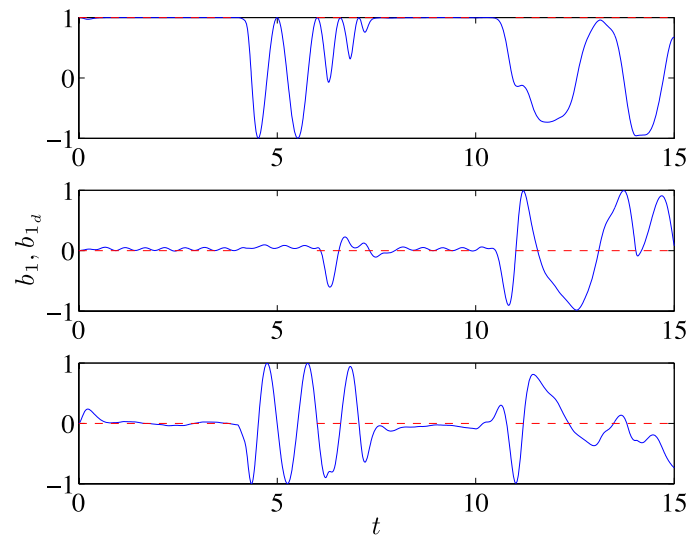

(e) First body-fixed axis $\left(b_{1}\right.$ :solid, $b_{1_{d}}$ :dotted)
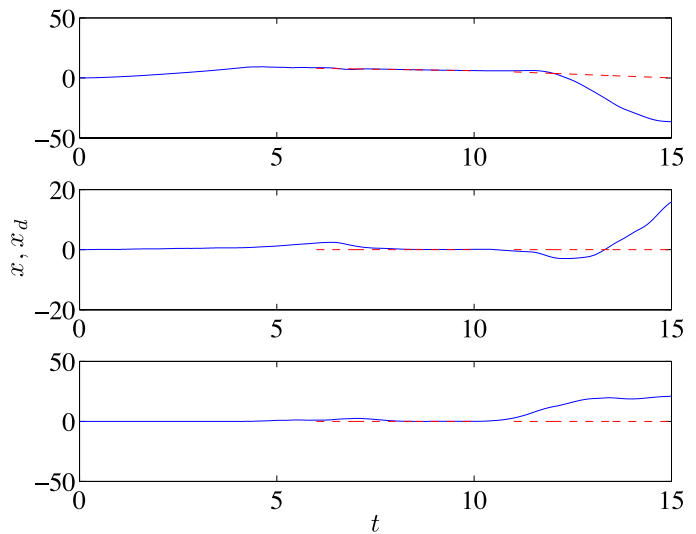

(b) Position ( $x$ :solid, $x_{d}$ :dotted, (m))
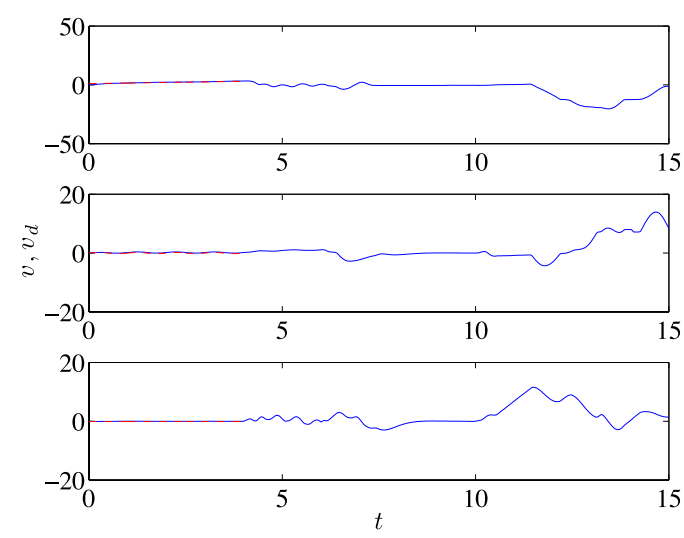

(d) Velocity ( $v$ :solid, $v_{d}$ :dotted, $(\mathrm{m} / \mathrm{s}))$

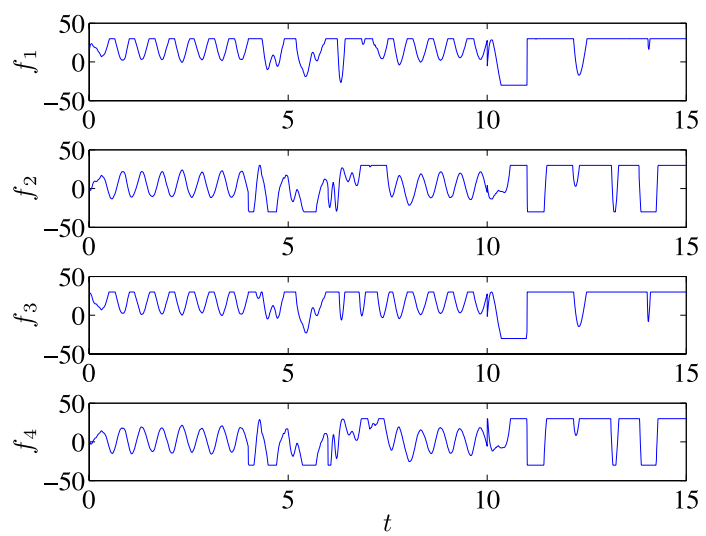

(f) Thrust of each rotor $(\mathrm{N})$

Fig. 8. Case II: transitions between several flight modes for a complex maneuver. The robust control input terms are set to zero, i.e. $\mu_{x}=\mu_{R}=0$, for comparison with Figure 7 . 
Lyapunov candidate. Let a Lyapunov candidate $\mathcal{V}_{2}$ be

$$
\mathcal{V}_{2}=\frac{1}{2} e_{\Omega} \cdot J e_{\Omega}+k_{R} \Psi\left(R, R_{d}\right)+c_{2} e_{R} \cdot e_{\Omega}
$$

We analyzes the properties of $\mathcal{V}_{2}$ along the solutions of the controlled system in the following domain $D_{2}$ :

$$
D_{2}=\left\{(R, \Omega) \in \mathrm{SO}(3) \times \mathbb{R}^{3} \mid \Psi\left(R, R_{d}\right)<\psi_{2}\right\} .
$$

From (11), (12), the attitude error function is bounded in $D_{2}$ as follows:

$$
\frac{1}{2}\left\|e_{R}\right\|^{2} \leq \Psi\left(R, R_{d}\right) \leq \frac{1}{2-\psi_{2}}\left\|e_{R}\right\|^{2},
$$

which implies that $\Psi$ is positive-definite and decrescent. It follows that the Lyapunov function $\mathcal{V}_{2}$ is bounded as

$$
z_{2}^{T} M_{21} z_{2} \leq \mathcal{V}_{2} \leq z_{2}^{T} M_{22} z_{2},
$$

where $z_{2}=\left[\left\|e_{R}\right\|,\left\|e_{\Omega}\right\|\right]^{T} \in \mathbb{R}^{2}$, and the matrices $M_{12}, M_{22}$ are given by

$$
M_{21}=\frac{1}{2}\left[\begin{array}{cc}
k_{R} & -c_{2} \\
-c_{2} & \lambda_{m}(J)
\end{array}\right], M_{22}=\frac{1}{2}\left[\begin{array}{cc}
\frac{2 k_{R}}{2-\psi_{2}} & c_{2} \\
c_{2} & \lambda_{M}(J)
\end{array}\right] .
$$

From (52), (53), (57), the time derivative of $\mathcal{V}_{2}$ along the solution of the controlled system is given by

$$
\begin{aligned}
\dot{\mathcal{V}}_{2}= & -k_{\Omega}\left\|e_{\Omega}\right\|^{2}-c_{2} k_{R} e_{R} \cdot J^{-1} e_{R}+c_{2} E\left(R, R_{d}\right) e_{\Omega} \cdot e_{\Omega} \\
& -c_{2} k_{\Omega} e_{R} \cdot J^{-1} e_{\Omega}+\left(e_{\Omega}+c_{2} J^{-1} e_{R}\right) \cdot\left(\Delta_{R}+\mu_{R}\right) .
\end{aligned}
$$

Since $\left\|E\left(R_{d}, R\right)\right\| \leq 1$, this is bounded by

$$
\dot{\mathcal{V}}_{2} \leq-z_{2}^{T} W_{2} z_{2}+e_{A} \cdot\left(\Delta_{R}+\mu_{R}\right),
$$

where $e_{A}=e_{\Omega}+c_{2} J^{-1} e_{R} \in \mathbb{R}^{3}$ and the matrix $W_{2} \in \mathbb{R}^{2 \times 2}$ is given by

$$
W_{2}=\left[\begin{array}{cc}
\frac{c_{2} k_{R}}{\lambda_{M}(J)} & -\frac{c_{2} k_{\Omega}}{2 \lambda_{m}(J)} \\
-\frac{c_{2} k_{\Omega}}{2 \lambda_{m}(J)} & k_{\Omega}-c_{2}
\end{array}\right] .
$$

Substituting (14), the last term of (64) is bounded by

$$
\begin{aligned}
e_{A} \cdot\left(\Delta_{R}+\mu_{R}\right) & =\delta_{R}\left\|e_{A}\right\|-\frac{\delta_{R}^{2}\left\|e_{A}\right\|^{2}}{\delta_{R}\left\|e_{A}\right\|+\varepsilon_{R}} \\
& =\varepsilon_{R} \frac{\delta_{R}\left\|e_{A}\right\|}{\delta_{R}\left\|e_{A}\right\|+\varepsilon_{R}} \leq \varepsilon_{R}
\end{aligned}
$$

to obtain

$$
\dot{\mathcal{V}}_{2} \leq-z_{2}^{T} W_{2} z_{2}+\varepsilon_{R}
$$

Boundedness. The condition (17) for the constant $c_{2}$ guarantees that the matrix $W_{2}$ in (66) and the matrices $M_{21}, M_{22}$ in (61) are positive-definite. Therefore, we obtain

$$
\begin{aligned}
& \lambda_{m}\left(M_{21}\right)\left\|z_{2}\right\|^{2} \leq \mathcal{V}_{2} \leq \lambda_{M}\left(M_{22}\right)\left\|z_{2}\right\|^{2}, \\
& \dot{\mathcal{V}}_{2} \leq-\lambda_{m}\left(W_{2}\right)\left\|z_{2}\right\|^{2}+\varepsilon_{R} .
\end{aligned}
$$

This implies that $\dot{\mathcal{V}}_{2}<0$ when

$$
\mathcal{V}_{2}>\left\{\frac{\lambda_{M}\left(M_{22}\right)}{\lambda_{m}\left(W_{2}\right)} \varepsilon_{R} \triangleq d_{1}\right\} .
$$

Consider a sub-level set of the Lyapunov function $\mathcal{V}_{2}$, defined as $\left.S_{\gamma}=\{R, \Omega\} \in \mathrm{SO}(3) \times \mathbb{R}^{3} \mid \mathcal{V}_{2} \leq \gamma\right\}$ for a positive constant $\gamma$. If $\gamma$ satisfies the following inequality

$$
\gamma<\left\{\lambda_{m}\left(M_{21}\right) \psi_{2}\left(2-\psi_{2}\right) \triangleq d_{2}\right\},
$$

then we can guarantee that $S_{\gamma}$ is a subset of the domain $D_{2}$ defined in (59).

In short, a sub-level set of the Lyapunov function, $S_{\gamma}$ is a positively invariant set when $d_{1}<\gamma<d_{2}$, and any solution starting in $S_{\gamma}$ exponentially converges to $S_{d_{1}}$. To guarantee the existence of such a set, we require

$$
\left\{d_{1}=\frac{\lambda_{M}\left(M_{22}\right)}{\lambda_{m}\left(W_{2}\right)} \varepsilon_{R}\right\}<\left\{\lambda_{m}\left(M_{21}\right) \psi_{2}\left(2-\psi_{2}\right) \triangleq d_{2}\right\},
$$

which can be achieved by requiring that (18) holds. Then, according to theorem 5.1 in [32], the attitude tracking errors are uniformly ultimately bounded, and the corresponding ultimate bound is estimated by

$$
S_{d_{1}} \subset\left\{\left\|z_{2}\right\|^{2} \leq \frac{\lambda_{M}\left(M_{22}\right)}{\lambda_{m}\left(M_{21}\right) \lambda_{m}\left(W_{2}\right)} \varepsilon_{R}\right\} .
$$

Furthermore, a sub-level set of the Lyapunov function $\mathcal{V}_{2}$ in $D_{2}$ corresponds to an estimate of the region of attraction.

\subsection{Proof of Proposition 3}

We first derive the tracking error dynamics and a Lyapunov function for the translational dynamics of a quadrotor $\mathrm{UAV}$, and later it is combined with the stability analyses of the rotational dynamics in the section Appendix 8.2 to guarantee the boundedness of tracking errors.

The subsequent analyses are developed in the domain $D_{1}$.

$$
\begin{aligned}
D_{1}= & \left\{\left(e_{x}, e_{v}, R, e_{\Omega}\right) \in \mathbb{R}^{3} \times \mathbb{R}^{3} \times \mathrm{SO}(3) \times \mathbb{R}^{3} \mid\right. \\
& \left.\left\|e_{x}\right\|<e_{x_{\max }}, \Psi<\psi_{1}\right\},
\end{aligned}
$$

Similar to (60), we can show that 


$$
\frac{1}{2}\left\|e_{R}\right\|^{2} \leq \Psi\left(R, R_{c}\right) \leq \frac{1}{2-\psi_{1}}\left\|e_{R}\right\|^{2}
$$

Translational error dynamics. The time derivative of the position error is $\dot{e}_{x}=e_{v}$. The time-derivative of the velocity error is given by

$$
m \dot{e}_{v}=m \ddot{x}-m \ddot{x}_{d}=m g e_{3}-f R e_{3}-m \ddot{x}_{d}+\Delta_{x} .
$$

Consider the quantity $e_{3}^{T} R_{c}^{T} R e_{3}$, which represents the cosine of the angle between $b_{3}=R e_{3}$ and $b_{c_{3}}=R_{c} e_{3}$. Since $1-\Psi\left(R, R_{c}\right)$ represents the cosine of the eigen-axis rotation angle between $R_{c}$ and $R$, we have $1>e_{3}^{T} R_{c}^{T} R e_{3}>1-\Psi\left(R, R_{c}\right)>0$ in $D_{1}$. Therefore, the quantity $\frac{1}{e_{3}^{T} R_{c}^{T} R e_{3}}$ is well-defined. To rewrite the error dynamics of $e_{v}$ in terms of the attitude error $e_{R}$, we add and subtract $\frac{f}{e_{3}^{T} R_{c}^{T} R e_{3}} R_{c} e_{3}$ to the right hand side of (71) to obtain

$$
m \dot{e}_{v}=m g e_{3}-m \ddot{x}_{d}-\frac{f}{e_{3}^{T} R_{c}^{T} R e_{3}} R_{c} e_{3}-X+\Delta_{x}
$$

where $X \in \mathbb{R}^{3}$ is defined by

$$
X=\frac{f}{e_{3}^{T} R_{c}^{T} R e_{3}}\left(\left(e_{3}^{T} R_{c}^{T} R e_{3}\right) R e_{3}-R_{c} e_{3}\right)
$$

Let $A=-k_{x} e_{x}-k_{v} e_{v}-m g e_{3}+m \ddot{x}_{d}+\mu_{x}$. Then, from (27), (24), we have $f=-A \cdot R e_{3}$ and $b_{3_{c}}=R_{c} e_{3}=-A /\|A\|$, i.e., $-A=\|A\| R_{c} e_{3}$. By combining these, we obtain $f=\left(\|A\| R_{c} e_{3}\right)$ $\cdot \operatorname{Re}_{3}$. Therefore, the third term of the right hand side of (72) can be written as

$$
\begin{aligned}
-\frac{f}{e_{3}^{T} R_{c}^{T} \operatorname{Re}_{3}} R_{c} e_{3} & =-\frac{\left(\|A\| R_{c} e_{3}\right) \cdot \operatorname{Re}_{3}}{e_{3}^{T} R_{c}^{T} R e_{3}} \cdot-\frac{A}{\|A\|}=A \\
& =-k_{x} e_{x}-k_{v} e_{v}-m g e_{3}+m \ddot{x}_{d}+\mu_{x} .
\end{aligned}
$$

Substituting this into (72), the error dynamics of $e_{v}$ can be written as

$$
m \dot{e}_{v}=-k_{x} e_{x}-k_{v} e_{v}-X+\Delta_{x}+\mu_{x}
$$

Lyapunov candidate for translation dynamics. Let a Lyapunov candidate $\mathcal{V}_{1}$ be

$$
\mathcal{V}_{1}=\frac{1}{2} k_{x}\left\|e_{x}\right\|^{2}+\frac{1}{2} m\left\|e_{v}\right\|^{2}+c_{1} e_{x} \cdot e_{v} .
$$

The derivative of $\mathcal{V}_{1}$ along the solution of (74) is given by

$$
\begin{aligned}
\dot{\mathcal{V}}_{1}= & -\left(k_{v}-c_{1}\right)\left\|e_{v}\right\|^{2}-\frac{c_{1} k_{x}}{m}\left\|e_{x}\right\|^{2}-\frac{c_{1} k_{v}}{m} e_{x} \cdot e_{v} \\
& +\left\{-X+\Delta_{x}+\mu_{x}\right\} \cdot\left\{\frac{c_{1}}{m} e_{x}+e_{v}\right\} .
\end{aligned}
$$

From (29), (30), the last part of (76) is bounded by

$$
\begin{aligned}
e_{B} \cdot\left(\Delta_{x}+\mu_{x}\right) & \leq \delta_{x}\left\|e_{B}\right\|-\frac{\delta_{x}^{\tau+2}\left\|e_{B}\right\|^{\tau+2}}{\delta_{x}^{\tau+1}\left\|e_{B}\right\|^{\tau+1}+\varepsilon_{x}^{\tau+1}} \\
& =\frac{\delta_{x}\left\|e_{B}\right\| \varepsilon_{x}^{\tau+1}}{\delta_{x}^{\tau+1}\left\|e_{B}\right\|^{\tau+1}+\varepsilon_{x}^{\tau+1}} \leq \varepsilon_{x} .
\end{aligned}
$$

The last inequality is satisfied, since if $\delta_{x}\left\|e_{B}\right\| \leq \varepsilon_{x}$

$$
\delta_{x}\left\|e_{B}\right\| \frac{\varepsilon_{x}^{\tau+1}}{\delta_{x}^{\tau+1}\left\|e_{B}\right\|^{\tau+1}+\varepsilon_{x}^{\tau+1}} \leq \delta_{x}\left\|e_{B}\right\| \leq \varepsilon_{x},
$$

and if $\delta_{x}\left\|e_{B}\right\|>\varepsilon_{x}$

$$
\frac{\delta_{x}^{\tau+1}\left\|e_{B}\right\|^{\tau+1}}{\delta_{x}^{\tau+1}\left\|e_{B}\right\|^{\tau+1}+\varepsilon_{x}^{\tau+1}} \frac{\varepsilon_{x}^{\tau+1}}{\left(\delta_{x}\left\|e_{B}\right\|\right)^{\tau}} \leq\left(\frac{\varepsilon_{x}}{\delta_{x}\left\|e_{B}\right\|}\right)^{\tau} \varepsilon_{x} \leq \varepsilon_{x} .
$$

Now, we find a bound for $X$, as defined in (73). Since $f=\|A\|\left(e_{3}^{T} R_{c}^{T} R e_{3}\right)$, we have

$$
\begin{aligned}
\|X\| \leq & \|A\|\left\|\left(e_{3}^{T} R_{c}^{T} R e_{3}\right) R e_{3}-R_{c} e_{3}\right\| \\
\leq & \left(k_{x}\left\|e_{x}\right\|+k_{v}\left\|e_{v}\right\|+B+\delta_{x}\right) \\
& \times\left\|\left(e_{3}^{T} R_{c}^{T} R e_{3}\right) R e_{3}-R_{c} e_{3}\right\| .
\end{aligned}
$$

The last term $\left\|\left(e_{3}^{T} R_{c}^{T} R e_{3}\right) R e_{3}-R_{c} e_{3}\right\|$ represents the sine of the angle between $b_{3}=R e_{3}$ and $b_{c_{3}}=R_{c} e_{3}$, since

$$
\left(b_{3_{c}} \cdot b_{3}\right) b_{3}-b_{3_{c}}=b_{3} \times\left(b_{3} \times b_{3_{c}}\right) .
$$

The magnitude of the attitude error vector, $\left\|e_{R}\right\|$ represents the sine of the eigen-axis rotation angle between $R_{c}$ and $R$ (see [18]). Therefore, we have $\left\|\left(e_{3}^{T} R_{c}^{T} R e_{3}\right) R e_{3}-R_{c} e_{3}\right\| \leq\left\|e_{R}\right\|$. It follows that

$$
\begin{aligned}
\left\|\left(e_{3}^{T} R_{d}^{T} R e_{3}\right) R e_{3}-R_{d} e_{3}\right\| & \leq\left\|e_{R}\right\|=\sqrt{\Psi(2-\Psi)} \\
& \leq\left\{\sqrt{\psi_{1}\left(2-\psi_{1}\right)} \triangleq \alpha\right\}<1 .
\end{aligned}
$$

Therefore, $X$ is bounded by

$$
\begin{aligned}
\|X\| & \leq\left(k_{x}\left\|e_{x}\right\|+k_{v}\left\|e_{v}\right\|+B+\delta_{x}\right)\left\|e_{R}\right\| \\
& \leq\left(k_{x}\left\|e_{x}\right\|+k_{v}\left\|e_{v}\right\|+B+\delta_{x}\right) \alpha .
\end{aligned}
$$

Substituting (77), (79) into (76), 


$$
\begin{aligned}
\dot{\mathcal{V}}_{1} \leq & -\left(k_{v}(1-\alpha)-c_{1}\right)\left\|e_{v}\right\|^{2}-\frac{c_{1} k_{x}}{m}(1-\alpha)\left\|e_{x}\right\|^{2} \\
& +\frac{c_{1} k_{v}}{m}(1+\alpha)\left\|e_{x}\right\|\left\|e_{v}\right\| \\
& +\left\|e_{R}\right\|\left\{\left(B+\delta_{x}\right)\left(\frac{c_{1}}{m}\left\|e_{x}\right\|+\left\|e_{v}\right\|\right)+k_{x}\left\|e_{x}\right\|\left\|e_{v}\right\|\right\} \\
& +\varepsilon_{x} .
\end{aligned}
$$

In the above expression for $\dot{\mathcal{V}}_{1}$, there is a third-order error term, namely $k_{x}\left\|e_{R}\right\|\left\|e_{x}\right\|\left\|e_{v}\right\|$. Using (78), it is possible to estimate it with an upper bound $k_{x} \alpha\left\|e_{x}\right\|\left\|e_{v}\right\|$, which is similar to the other terms, but the corresponding stability analysis becomes complicated, and in practice, the initial attitude error needs to be reduced further. Instead, we restrict our analysis to the domain $D_{1}$ defined in (69), and its upper bound is chosen as $k_{x} e_{x_{\max }}\left\|e_{R}\right\|\left\|e_{v}\right\|$.

Lyapunov candidate for the complete system. Let $\mathcal{V}=$ $\mathcal{V}_{1}+\mathcal{V}_{2}$ be the Lyapunov candidate of the complete system.

$$
\begin{aligned}
\mathcal{V}= & \frac{1}{2} k_{x}\left\|e_{x}\right\|^{2}+\frac{1}{2} m\left\|e_{v}\right\|^{2}+c_{1} e_{x} \cdot e_{v} \\
& +\frac{1}{2} e_{\Omega} \cdot J e_{\Omega}+k_{R} \Psi\left(R, R_{d}\right)+c_{2} e_{R} \cdot e_{\Omega} .
\end{aligned}
$$

Using (70), the bound of the Lyapunov candidate $\mathcal{V}$ can be written as

$$
z_{1}^{T} M_{11} z_{1}+z_{2}^{T} M_{21} z_{2} \leq \mathcal{V} \leq z_{1}^{T} M_{12} z_{1}+z_{2}^{T} M_{22}^{\prime} z_{2},
$$

where $z_{1}=\left[\left\|e_{x}\right\|, \quad\left\|e_{v}\right\|\right]^{T}, \quad z_{2}=\left[\left\|e_{R}\right\|, \quad\left\|e_{\Omega}\right\|\right]^{T} \in \mathbb{R}^{2}$, and the matrices $M_{11}, M_{12}, M_{21}, M_{22}$ are given by

$$
\begin{aligned}
& M_{11}=\frac{1}{2}\left[\begin{array}{cc}
k_{x} & -c_{1} \\
-c_{1} & m
\end{array}\right], \quad M_{12}=\frac{1}{2}\left[\begin{array}{cc}
k_{x} & c_{1} \\
c_{1} & m
\end{array}\right], \\
& M_{21}=\frac{1}{2}\left[\begin{array}{cc}
k_{R} & -c_{2} \\
-c_{2} & \lambda_{m}(J)
\end{array}\right], \quad M_{22}^{\prime}=\frac{1}{2}\left[\begin{array}{cc}
\frac{2 k_{R}}{2-\psi_{1}} & c_{2} \\
c_{2} & \lambda_{M}(J)
\end{array}\right] .
\end{aligned}
$$

Using (66) and (80), the time-derivative of $\mathcal{V}$ is given by

$$
\dot{\mathcal{V}} \leq-z_{1}^{T} W_{1} z_{1}+z_{1}^{T} W_{12} z_{2}-z_{2}^{T} W_{2} z_{2}+\varepsilon_{x}+\varepsilon_{R}
$$

where $W_{1}, W_{12}, W_{2} \in \mathbb{R}^{2 \times 2}$ are defined as follows:

$$
\begin{aligned}
& W_{1}=\left[\begin{array}{cc}
\frac{c_{1} k_{x}}{m}(1-\alpha) & -\frac{c_{1} k_{v}}{2 m}(1+\alpha) \\
-\frac{c_{1} k_{v}}{2 m}(1+\alpha) & k_{v}(1-\alpha)-c_{1}
\end{array}\right], \\
& W_{12}=\left[\begin{array}{cc}
\frac{c_{1}}{m}\left(B+\delta_{x}\right) & 0 \\
B+\delta_{x}+k_{x} e_{x_{\max }} & 0
\end{array}\right],
\end{aligned}
$$

$$
W_{2}=\left[\begin{array}{cc}
\frac{c_{2} k_{R}}{\lambda_{M}(J)} & -\frac{c_{2} k_{\Omega}}{2 \lambda_{m}(J)} \\
-\frac{c_{2} k_{\Omega}}{2 \lambda_{m}(J)} & k_{\Omega}-c_{2}
\end{array}\right] .
$$

Boundedness. Under the given conditions (35), (36), all of the matrices $M_{11}, M_{12}, M_{21}, M_{22}^{\prime}, W_{1}$, and $W_{2}$ are positivedefinite. Therefore, the Lyapunov function $\mathcal{V}$ is positivedefinite and decrescent, so we obtain

$$
\begin{aligned}
& \min \left\{\lambda_{m}\left(M_{11}\right), \lambda_{m}\left(M_{21}\right)\right\}\|z\|^{2} \leq \mathcal{V} \\
& \quad \leq \max \left\{\lambda_{M}\left(M_{12}\right), \lambda_{M}\left(M_{22}^{\prime}\right)\right\}\|z\|^{2},
\end{aligned}
$$

where $z=\left[\left\|z_{1}\right\|,\left\|z_{2}\right\|\right]^{T} \in \mathbb{R}^{2}$, and the time-derivative of $\mathcal{V}$ is bounded by

$$
\begin{aligned}
\dot{\mathcal{V}} \leq & -\lambda_{m}\left(W_{1}\right)\left\|z_{1}\right\|^{2}+\left\|W_{12}\right\|_{2}\left\|z_{1}\right\|\left\|z_{2}\right\|-\lambda_{m}\left(W_{2}\right)\left\|z_{2}\right\|^{2} \\
& +\varepsilon_{x}+\varepsilon_{R} \\
= & -z^{T} W z+\varepsilon_{x}+\varepsilon_{R} \\
\leq & -\lambda_{m}(W)\|z\|^{2}+\varepsilon_{x}+\varepsilon_{R} .
\end{aligned}
$$

where the matrix $W \in \mathbb{R}^{2 \times 2}$ is given by

$$
W=\left[\begin{array}{cc}
\lambda_{m}\left(W_{1}\right) & -\frac{1}{2}\left\|W_{12}\right\|_{2} \\
-\frac{1}{2}\left\|W_{12}\right\|_{2} & \lambda_{m}\left(W_{2}\right)
\end{array}\right] .
$$

Similar to the proof of Proposition 2, we can show that the tracking errors are uniformly ultimately bounded if the constants $\varepsilon_{x}, \varepsilon_{R}$ are sufficiently small, as given in (38), and the corresponding ultimate bound is given by (39). A sub-level set of the Lyapunov function $\mathcal{V}$ contained in the domain $D_{1}$ is an estimate to the region of attraction.

\subsection{Proof of Proposition 4}

The given assumptions satisfy the assumptions of Proposition 2, from which the tracking error $z_{2}=\left[\left\|e_{R}\right\|,\left\|e_{\Omega}\right\|\right]^{T}$ is guaranteed to exponentially decrease until it satisfies the bound given by (19). But, (42) guarantees that the attitude error enters the region defined by (33) in a finite time $t^{*}$.

Therefore, if we show that the tracking error $z_{1}=\left[\left\|e_{x}\right\|\right.$, $\left.\left\|e_{v}\right\|\right]^{T}$ is bounded in $t \in\left[0, t^{*}\right]$ as well, then the complete tracking error $\left(z_{1}, z_{2}\right)$ is uniformly ultimately bounded.

The boundedness of $z_{1}$ is shown as follows. The error dynamics or $e_{v}$ can be written as

$$
m \dot{e}_{v}=m g e_{3}-f R e_{3}-m \ddot{x}_{d}+\Delta_{x} .
$$

Let $\mathcal{V}_{3}$ be a positive-definite function of $\left\|e_{x}\right\|$ and $\left\|e_{v}\right\|$ : 


$$
\mathcal{V}_{3}=\frac{1}{2}\left\|e_{x}\right\|^{2}+\frac{1}{2} m\left\|e_{v}\right\|^{2}
$$

Then, we have $\left\|e_{x}\right\| \leq \sqrt{2 \mathcal{V}_{3}},\left\|e_{v}\right\| \leq \sqrt{\frac{2}{m} \mathcal{V}_{3}}$. The time-derivative of $\mathcal{V}_{3}$ is given by

$$
\begin{aligned}
\dot{\mathcal{V}}_{3} & =e_{x} \cdot e_{v}+e_{v} \cdot\left(m g e_{3}-f R e_{3}-m \ddot{x}_{d}+\Delta_{x}\right) \\
& \leq\left\|e_{x}\right\|\left\|e_{v}\right\|+\left\|e_{v}\right\|\left(B+\delta_{x}\right)+\left\|e_{v}\right\|\left\|R e_{3}\right\| f \mid .
\end{aligned}
$$

From (27), we obtain

$$
\begin{aligned}
\dot{\mathcal{V}}_{3} \leq & \left\|e_{x}\right\|\left\|e_{v}\right\|+\left\|e_{v}\right\|\left(B+\delta_{x}\right) \\
& +\left\|e_{v}\right\|\left(k_{x}\left\|e_{x}\right\|+k_{v}\left\|e_{v}\right\|+B+\delta_{x}\right) \\
= & k_{v}\left\|e_{v}\right\|^{2}+\left(2\left(B+\delta_{x}\right)+\left(k_{x}+1\right)\left\|e_{x}\right\|\right)\left\|e_{v}\right\| \\
\leq & d_{1} \mathcal{V}_{3}+d_{2} \sqrt{\mathcal{V}_{3}},
\end{aligned}
$$

where $d_{1}=k_{v} \frac{2}{m}+2\left(k_{x}+1\right) \frac{1}{\sqrt{m}}, d_{2}=2\left(B+\delta_{x}\right) \sqrt{\frac{2}{m}}$. Suppose that $\mathcal{V}_{3} \geq 1$ for a time interval $\left[t_{a}, t_{b}\right] \subset\left[0, t^{*}\right]$. In this time interval, we have $\sqrt{\mathcal{V}_{3}} \leq \mathcal{V}_{3}$. Therefore,

$$
\dot{\mathcal{V}}_{3} \leq\left(d_{1}+d_{2}\right) \mathcal{V}_{3} \Rightarrow \mathcal{V}_{3}(t) \leq \mathcal{V}_{3}\left(t_{a}\right) e^{\left(d_{1}+d_{2}\right)\left(t-t_{a}\right)}
$$

Therefore, for any time interval in which $\mathcal{V}_{3} \geq 1, \mathcal{V}_{3}$ is bounded. This implies that $\mathcal{V}_{3}$, and therefore $z_{1}=\left[\left\|e_{x}\right\|,\left\|e_{v}\right\|\right]^{T}$, are bounded for $0 \leq t \leq t^{*}$.

\section{REFERENCES}

1. Valenti, M., B. Bethke, G. Fiore, and J. How, "Indoor multi-vehicle flight testbed for fault detection, isolation, and recovery," in Proceedings of the AIAA Guidance, Navigation and Control Conference, AIAA-2006-6200 (2006).

2. Hoffmann, G., H. Huang, S. Waslander, and C. Tomlin, "Quadrotor helicopter flight dynamics and control: Theory and experiment," in Proceedings of the AIAA Guidance, Navigation, and Control Conference, AIAA 2007-6461 (2007).

3. Castillo, P., R. Lozano, and A. Dzul, "Stabilization of a mini rotorcraft with four rotors," IEEE Control Syst. Mag., Vol. 3, No. 6, pp. 45-55 (2005).

4. Bouabdalla, S. and R. Siegward, "Backstepping and sliding-mode techniques applied to an indoor micro quadrotor," in Proc. IEEE Int Conf. Rob. Autom., pp. 2259-2264 (2005).

5. Efe, M., "Robust low altitude behavior control of a quadrotor rotorcraft through sliding modes," in Proceedings of the Mediterranean Conference on Control and Automation, pp. 1-6 (2007).

6. Raffo, G., M. Ortega, and F. Rubio, "An integral predictive/nonlinear $H_{\infty}$ control structure for a quadrotor helicopter," Automatica, Vol. 46, No. 1, pp. 29-39 (2010).
7. Nicol, C., C. Macnab, and A. Ramirez-Serrano, "Robust neural network control of a quadrotor helicopter," in Proceedings of the Canadian Conference on Electrical and Computer Engineering, pp. 1233-1237 (2008).

8. Tayebi, A. and S. McGilvray, "Attitude stabilization of a VTOL quadrotor aircraft," IEEE Trans. Control Syst. Technol., Vol. 14, No. 3, pp. 562-571 (2006).

9. Mayhew, C., R. Sanfelice, and A. Teel, "Quaternionbased hybrid control for robust global attitude tracking," IEEE Trans. Autom. Control., Vol. 56, No. 11, pp. 25552566 (2011).

10. Mayhew, C., R. Sanfelice, and A. Teel, "On the nonrobustness of inconsistent quaternion-based attitude control systems using memoryless path-lifting schemes," in Proc. Amer. Control Conf. (2011).

11. Bhat, S. and D. Bernstein, "A topological obstruction to continuous global stabilization of rotational motion and the unwinding phenomenon," Syst. Control Lett., Vol. 39, No. 1, pp. 66-73 (2000).

12. Mayhew, C. R. Sanfelice, and A. Teel, "On quaternionbased attitude control and the unwinding phenomenon," in Proc. Amer. Control Conf., pp. 299-304 (2011).

13. Bullo, F. and A. Lewis, Geometric control of mechanical systems, ser. Texts in Applied Mathematics. New York: Springer-Verlag, Vol. 49, modeling, analysis, and design for simple mechanical control systems (2005).

14. Maithripala, D., J. Berg, and W. Dayawansa, "Almost global tracking of simple mechanical systems on a general class of Lie groups," IEEE Trans. Autom. Control., Vol. 51, No. 1, pp. 216-225 (2006).

15. Cabecinhas, D., R. Cunha, and C. Silvestre, "Outputfeedback control for almost global stabilization of fully-acuated rigid bodies," in Proceedings of IEEE Conference on Decision and Control, 3583-3588, Ed., (2008).

16. Chaturvedi, N., A. Sanyal, and N. McClamroch, "Rigidbody attitude control," IEEE Control Syst. Mag., Vol. 31, No. 3, pp. 30-51 (2011).

17. Lee, T., M. Leok, and N. McClamroch, "Geometric tracking control of a quadrotor UAV on SE(3)," in Proceedings of the IEEE Conference on Decision and Control, pp. 5420-5425 (2010).

18. Lee, T., M. Leok, and N. McClamroch, "Control of complex maneuvers for a quadrotor UAV using geometric methods on $\operatorname{SE}(3)$," arXiv. [Online]. Available: http:// arxiv.org/abs/1003.2005

19. Cabecinhas, D., R. Cunha, and C. Silvestre, "Rotorcraft path following control for extended flight envelope coverage," in Proceedings of the IEEE Conference on Decision and Control, pp. 3460-3465 (2009).

20. Mellinger, D. and V. Kumar, "Minimum snap trajectory generation and control for quadrotors," in Proceedings of the International Conference on Robotics and Automation (2011). 
21. Naldi, R., L. Marconi, and L. Gentili, "Robust takeoff and landing for a class of aerial robots," in Proceedings of the IEEE Conference on Decision and Control, pp. 3436-3441 (2009).

22. Hua, M., T. Hamel, P. Morin, and C. Samson, "A control approach for thrust-propelled underactuated vehicles and its application to VTOL drones," IEEE Trans. Autom. Control., Vol. 54, No. 8, pp. 1834-1853 (2009).

23. Mellinger, D., N. Michael, and V. Kumar, "Trajectory generation and control for precise aggressive maneuvers with quadrotors," Int. J. Robot. Res., Vol. 31, No. 5, pp. 664-674 (2012).

24. Gillula, J., G. Hoffmann, H. Huang, M. Vitus, and C. Tomlin, "Applications of hybrid reachability analysis to robotic aerial vehicles," Int. J. Robust Nonlinear Control, Vol. 30, No. 3, pp. 335-354 (2011).

25. Oishi, M. and C. Tomlin, "Switched nonlinear control of a VSTOL aircraft," in Proceedings of IEEE Conference on Decision and Control, pp. 2685-2690 (1999).

26. Ghosh, R. and C. Tomlin, "Nonlinear inverse dynamic control for mode-based flight," in Proceedings of the AIAA Guidance, Navigation and Control Conference (2000).

27. Oishi, M. and C. Tomlin, "Switching in nonlinear minimum phase systems: Applications to a VSTOL aircraft," in Proc. Amer. Control Conf., pp. 487-491 (2000).

28. Frazzoli, E., M. Dahleh, and E. Feron, "Maneuver based motion planning for nonlinear systems with symmetries," IEEE Trans. Robot., Vol. 21, No. 6, pp. 1077-1091 (2005).

29. Chaturvedi, N., N. H. McClamroch, and D. Bernstein, "Asymptotic smooth stabilization of the inverted 3-D pendulum," IEEE Trans. Autom. Control., Vol. 54, No. 6, pp. 1204-1215 (2009).

30. Lee, T., "Robust adaptive geometric tracking controls on $\mathrm{SO}(3)$ with an application to the attitude dynamics of a quadrotor UAV," arXiv, 2011. [Online]. Available: http:// arxiv.org/abs/1108.6031

31. Pounds, P., R. Mahony, and P. Corke, "Modeling and control of a large quadrotor robot," Control Eng. Practice, Vol. 18, pp. 691-699 (2010).
32. Khalil, H. Nonlinear Systems, 2nd Edition, Ed. Prentice Hall (1996).

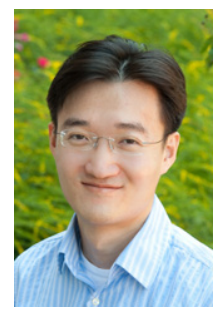

Taeyoung Lee is Assistant Professor of Department of Mechanical and Aerospace Engineering at George Washington University. He received his doctoral degree in Aerospace Engineering and his master's degree in Mathematics at University of Michigan in 2008. His research interests include computational geometric mechanics and control of complex systems.

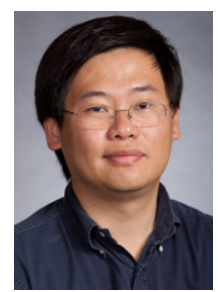

Melvin Leok is Associate Professor of Mathematics at University of California, San Diego. His research interests include computational aspects of geometric mechanics and geometric control theory, discrete geometry, and structure-preserving numerical schemes. He was the recipient of a NSF CAREER Award, SciCADE New Talent Prize, Leslie Fox Prize, and SIAM Student Paper Prize. He serves on the editorial boards of Journal of Nonlinear Science, SIAM Journal on Control and Optimization, LMS Journal of Computation and Mathematics, Journal of Geometric Mechanics, and Journal of Computational Dynamics.

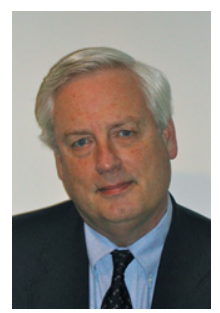

Harris McClamroch has been a faculty member at University of Michigan since 1967. He was Professor and past Chair of Department of Aerospace Engineering. He is currently Professor Emeritus of Aerospace Engineering. He has published more than 250 papers, most in collaboration with his twenty-seven PhD students, on theoretical control problems and control applications arising in robotics, manufacturing, buildings and bridges, and aerospace vehicles. He is Fellow of the IEEE and a recipient of several IEEE awards. He served as Editor of IEEE Transactions on Automatic Control, and he was President of IEEE Control Systems Society in 1998. 